\title{
Laser-Induced Plasma optical emission: from atomic to molecular spectra
}

\author{
Alessandro De Giacomo ${ }^{1}$ and Jörg Hermann ${ }^{2}$ \\ 1. Department of Chemistry, University of Bari, Via Orabona 4, 70125 Bari-Italy \\ Email: alessandro.degiacomo@uniba.it \\ 2. Aix-Marseille University, CNRS, LP3, 13288 Marseille, France \\ Email: jorg.hermann@cnrs.fr
}

\begin{abstract}
.
The aim of this paper is the description of the optical emission spectral features of the plasma produced by laser matter interaction from a fundamental point of view. The laser induced plasma emission spectra are discussed in connection with the basic mechanisms that take place in the plasma phase at different time delays from the laser pulse. Being the laser induced plasma a dynamics system, the hierarchy of the elementary mechanisms changes continuously because the electron number density and the electron temperature decrease during the expansion. As a consequence of this, along the plasma's persistence time, the prevailing emitting species changes from ions to atoms and from atoms to molecules. Both atomic and molecular emission spectroscopy are discussed to convey a complete description of temporal evolution of the laser induced plasma.

Current literature, as well as the traditional plasma's theories, are presented and discussed in order to give to the reader a general idea of the potentialities and of the drawbacks of emission spectroscopy for the study of laser induced plasma and the of the various applications.
\end{abstract}

\section{Introduction.}

Laser Induced Plasma (LIP) is the plasma generated by laser-matter interaction when the laser irradiance exceeds a certain threshold that is a characteristic of any specific materials.

The emission spectra of LIP have got a great interest in the scientific community because they allow to study the LIP's fundamental aspects and in turn to optimize the specific applications where the LIP is used. LIP is indeed applied to many different fields of science and among the most important applications there are: Pulsed Laser Deposition, Laser welding and cutting, Laser propulsion, Laser ablation in liquid for nanostructure production, Laser Induced Breakdown Spectroscopy (LIBS) and so on [1].

LIP, being an expanding plasma, changes its parameters fast during its evolution. Immediately after the ablation, the plasma has a number density close to that one irradiated sample and then expands at supersonic velocity, compressing the surrounding environment and generating an external shockwave [2]. When the pressure of the LIP is similar to that one of the internal wall of the shockwave, the expansion stops and the plasma is confined in the microenvironment limited by the shockwave wall [3-5]. In this simple view, it is clear that the dynamics of the LIP strongly depends by the external environment where the LIP is expanding such as state of matter (gas or liquid), chemical composition and pressure [6]. In the frame of this observation it is not easy to generalize the LIP characteristics by the temporal coordinates without specifying the experimental conditions. In any case it can be supposed that immediately after the laser pulse the plasma experiences extreme conditions in terms of 
temperature and number density of species. At these conditions the plasma is mainly constituted of ions, atoms and electrons. During the expansion the temperature decreases, inducing recombination and decreasing the number of charged particles in the plasma. When and where the temperature is sufficiently low the collisions between heavy particles (atoms and ions) can not be neglected any more with respect to electronic collisions and the formation of molecular species occurs [1,3,7]. Although the dynamics may be very different at different experimental conditions, we can generalize this route of the LIP where it is observable this succession of prevailing species from ions to atoms and from atoms to molecules. This turnover of the prevailing species is usually observed by the emission spectroscopy and is the weave of this review, where the interpretation of the spectral features of the LIP and their connection with the fundamental plasma processes are discussed.

Moreover, in this review, we discuss mainly the nanosecond laser plasma, because the interaction of the beam with the vaporized material leads to a larger degree of atomization and excitation and so it is more interesting from the spectroscopic point of view. The spectroscopy of ultrashort laser produced plasmas is uniquely used for micro-analysis, when a high spatial resolution is required. In any case, the present discussion is related to the laser-induced plasma after the ablation and plasma laser excitation is over. In this view, a detailed description of the effect of the laser pulse on the ablation and plasma excitation it would be out of scope and interested reader can refer to Refs. [2, 6] and references therein. Briefly the effect of laser pulse, in terms of deposited energy can be understood with the point explosion model of Sedov. This model, that describes well the initial fast expansion stage, considers the laser absorbed energy in a point at $t=0$. Thus, it makes in principal no difference if one uses nanosecond or ultrashort laser pulses for the study of the plasma emission techniques and the interpretation of the correlation between the spectral features and the basic mechanisms. The difference comes only from the laser interaction with the vaporized material in the nanosecond case, because higher excitation can be obtained as a consequence of laser absorption from the free electrons and photoionization. Also, the lifetime of the plasma depends mostly on the amount of deposited laser energy. As the laser pulse energy of ultrashort lasers is typically much smaller than that of ns laser pulses, the plasma lifetime is shorter for ultrashort laser ablation, and molecules are observed much earlier.

In any case, the optical emission of LIP allows to estimating plasma parameters such as electron number density, excitation temperature, ionization degree, plasma expansion rate and plasma composition [8,9]. The most of the studies based on LIP optical emission are focused on atomic spectroscopy because the atomic plasma is directly connected to the irradiated sample and the atomic emission is very intense as a consequence of the high number density of emitters and of the high temperature of the plasma. As an example the velocity of the species of the plasma has been studied during Pulsed Laser Deposition by detecting the temporal profile of atomic and ionic emission lines at different distances [10]. These kind of studies allow to understand the initial kinetic energy of the species and also allow to understand the question of the different velocity of species in the plasma. These investigations allow to optimize the adhesion on a substrate and the formation of thin films during laser deposition [11,12]. Traditionally the temperature of the plasma, obtained by emission spectroscopy, has been used as a quality check of laser welding process [13]. Moreover the temperature of the plasma in connection with the velocity of the ablated species has been investigated in order to improve the laser propulsion in space applications [14]. In particular the possibility of applying LIP emission spectroscopy for the determination of plasma composition, namely LIBS, allows to perform elementary analysis with the following advantages: fast response, no or minimal sample preparation, simultaneous multi-elemental detection of major and trace elements, capability for microanalysis with low sample amounts, standardless analysis etc [15-17]. In the frame of this technique the most of the elements spectra and their behaviors in the laser plasma have been studied [17]. Between the most common application of elemental analysis by LIBS we find environmental 
analysis [18], cultural heritage [19], sorting of objects in industrial applications [20], analysis of liquid samples [21], elemental mapping [22], space exploration [23], biological and medical applications [21, 22]. The success of LIBS in these applications is that it allows immediate elemental analysis with a very flexible methodology. It is important to underline that, for a successful use of LIBS in any application, it is necessary to deal with the fundamental aspects of the LIP, because, being the LIP a transient plasma, the optimization of the detection time and optical geometry of the acquisition system play a crucial role $[15,17]$. Although the most of the studies and application of emission spectroscopy of LIP concern the atomic optical emission, molecular features have attracted increasing interest in the diagnostics of laser-produced plasmas during the last years. The early studies were mainly dedicated to the understanding of chemical processes in thin film synthesis via pulsed laser deposition (PLD). In reactive PLD, a pure sample is ablated in a low-pressure background gas to deposit a composite formed by chemical reactions between the sample material and the gas [24]. Here, the role of gasphase reactions was investigated by observing molecular emission via time- and space-resolved optical emission spectroscopy [25]. More recently, the same approach was used to probe chemical processes in the synthesis of nanoparticles via laser ablation in liquids. In that case, the observed molecules or radicals are precursors in the nanoparticle formation process [26]. In analytical science, laser ablation molecular isotopic spectrometry (LAMIS) was introduced by Russo and coworkers [27] to measure isotopic ratios in materials. The method explores the large isotopic shift of molecular bands that is typically more than one order of magnitude larger than the isotopic shift of atomic lines [28].

In elemental analysis of materials via laser-induced breakdown spectroscopy (LIBS), the formation of molecular species was mostly considered to be a drawback: chemical reactions may lead to nonlinear calibration curves, and interferences of molecular bands with analytical lines may prevent from accurate line intensity measurements [29, 30]. However, some authors investigate molecular emissions in order to detect elements that usually pose challenges [31]. Thus, it was shown that the limits of halogen detection $(\mathrm{F}, \mathrm{Cl}, \ldots)$ are considerably reduced when observing the emission bands of their radicals formed by recombination with $\mathrm{Cu}$ or $\mathrm{Ca}$, instead of measuring the halogen atomic lines [31-33]. This enabled the detection of fluorine on planet Mars [34]. The exploration of molecular emission was also proposed to characterize combustion processes [35]. In LIBS analysis under water, the low-temperature and high density favor the plasma reactivity, and molecular emission bands present a useful tool for diagnostics [36].

The most prominent exploration of molecular emission in LIBS is related to the analysis of organic materials that has vast field of applications such as polymer recycling [37], detection of explosives [38], and food security [39]. Accordingly, the mostly observed diatomic species in laserproduced plasmas are $\mathrm{CN}, \mathrm{C}_{2}$ and other molecules or radicals formed by recombination between $\mathrm{C}, \mathrm{N}$, $\mathrm{H}$ and $\mathrm{O}$ [40-43]. Metal oxides were also currently reported [44-47]. Most of these observations concern $\mathrm{AlO}$ [48-50]. Beside $\mathrm{CN}$, the diatomic nitrides were rarely reported [25, 30, 51], although nitrogen is abundant in the ambient air.

The aim of this review is to discuss the fundamental aspects of LIP and the consequent effects on the spectral features of plasma species from the atomic to molecular spectra.

\section{Elementary processes in LIP and Thermodynamic classification.}

In the time interval between the laser pulse and several microseconds, LIP can be considered an atomic plasma as a consequence of the high temperature (tens of thousands of Kelvin) and high electron number density $\left(>10^{17} \mathrm{~cm}^{-3}\right)$. In this time interval LIP is dominated by electron impact 
processes such as elastic collisions between electrons and inelastic collisions between electrons and heavy particles (atoms and ions). Radiative processes are also observable and their role becomes important in the energy balance only when electron collision frequency decreases during the expansion stage $[6,42]$.

Let's consider the LIP as an ideal plasma obeying Maxwell-Boltzmann statistics and the following mechanisms [52]:

a) Elastic collision between electrons

$$
e^{-}\left(E_{e_{\alpha}^{-}}\right)+e^{-}\left(E_{e_{\beta}^{-}}\right) \Leftrightarrow e^{-}\left(E_{e_{\alpha}^{-}}-\Delta E_{\alpha \beta}\right)+e^{-}\left(E_{e_{\beta}^{-}}+\Delta E_{\alpha \beta}\right)
$$

b) Excitation de-excitation due to inelastic collision between electron and heavy particle:

$$
A_{l}+e^{-}\left(E_{e^{-}}\right) \underset{K^{\text {deexc }}}{\stackrel{K^{e x c}}{\Leftrightarrow}} A_{u}+e^{-}\left(E_{e^{-}}-\Delta E_{u l}\right)
$$

c) Ionization/recombination processes

$$
\begin{gathered}
A_{l}+e^{-}\left(E_{e^{-}}\right) \underset{K^{3 b}}{\stackrel{K^{i o n}}{\Leftrightarrow}} A^{+}+e^{-}\left(E_{e^{-}}-E_{e^{-}}^{\prime}-E_{i o n}+E_{l}\right)+e^{-}\left(E_{e^{-}}^{\prime}\right) \\
A_{l}+h v_{\left(E_{e^{-}}-E_{l}\right)} \underset{K^{R R}}{\stackrel{K^{h v}}{\Leftrightarrow}} A^{+}+e^{-}{ }_{\left(E_{e^{-}}-E_{i o n}+E_{l}\right)}
\end{gathered}
$$

d) Spontaneous emission and absorption

$$
A_{u} \Leftrightarrow A_{l}+h v_{u l}
$$

In the eqs. 1-4 $\mathrm{e}^{-}$and $A$ are electrons and heavy particles (atoms or ions) respectively and the subscripts $l$ and $u$ indicate the lower energy level and the upper energy level.

Elastic collisions between electrons are very efficient at typical LIP condition and, although the system may be perturbed as a consequence of the fast expansion and recombination processes, the 
electron energy distribution function (eedf) holds a Maxwell form for the most of the persisting time of the LIP $[52,53]$. The Maxwellian form of eedf suggests the possibility of observing the equilibrium between all material particles i.e. Local Thermal Equilibrium (LTE) condition. This condition implies that inelastic collisions between the fast electrons and the heavy particles, leading to heavy particle excitation, and the backward process of superelastic collisions, where the slow electrons gain energy from a collision with an excited heavy particles leading to de-excitation of the latter ones, are completely balanced. At this condition all heavy particles belong to a Boltzmann distribution with the same temperature of the eedf Maxwell distribution [3, 8, 46, 52]. In addition to excitation and deexcitation of heavy particles, electron collisions are also responsible of the production and destruction of charged particles with two possible mechanisms: electron impact ionization and three body recombination (eq.3a) and photo-ionization and radiative recombination (eq.3b). The latter one plays important role only during the laser pulse and at the beginning of the expansion and will be discussed in section 4, while the electron impact ionization and three body recombination is the main mechanism determining the ionization degree of the plasma for the most of the expansion [53, 54]. If the time associated to the de-excitation process by superelastic collision is less or comparable to the inverse of spontaneous emission coefficient of a given transition, the particle de-excitation can occur by spontaneous emission (eq.4). It is interesting to note that an expanding plasma is always followed by radiation emission as a consequence of the thermodynamic criterion of spontaneity (entropy $\Delta S>0$ ), so that it would be more appropriate to consider the plasma in near LTE conditions [55]. In this connection we assume that the energy loss by radiative decay of the heavy particle system is extremely little with respect of the energy exchanged with collisions and so the distribution function still hold a Boltzmann form $[52,53,56]$.

By this simplified description of the LIP processes, it is clear that the number of electrons plays a crucial role in supporting the plasma and in determining its properties. At the same time it is important to take in mind that LIP is a transient plasma where all the energy is delivered during the laser pulse and then the system evolves to the equilibrium with the external ambient environment. That means that, as a consequence of the expansion and of the exchange of energy with the surrounding environment, the electron number density and the excitation temperature strongly decrease. In this view the hierarchy of the collisional mechanisms and the thermodynamic properties of the plasma change continuously along the persistence time [46]. In Fig.1 it is shown, as an example, the electron number density evolution and the excitation temperature calculated with Stark broadening [8] and the Boltzmann plot technique respectively. 


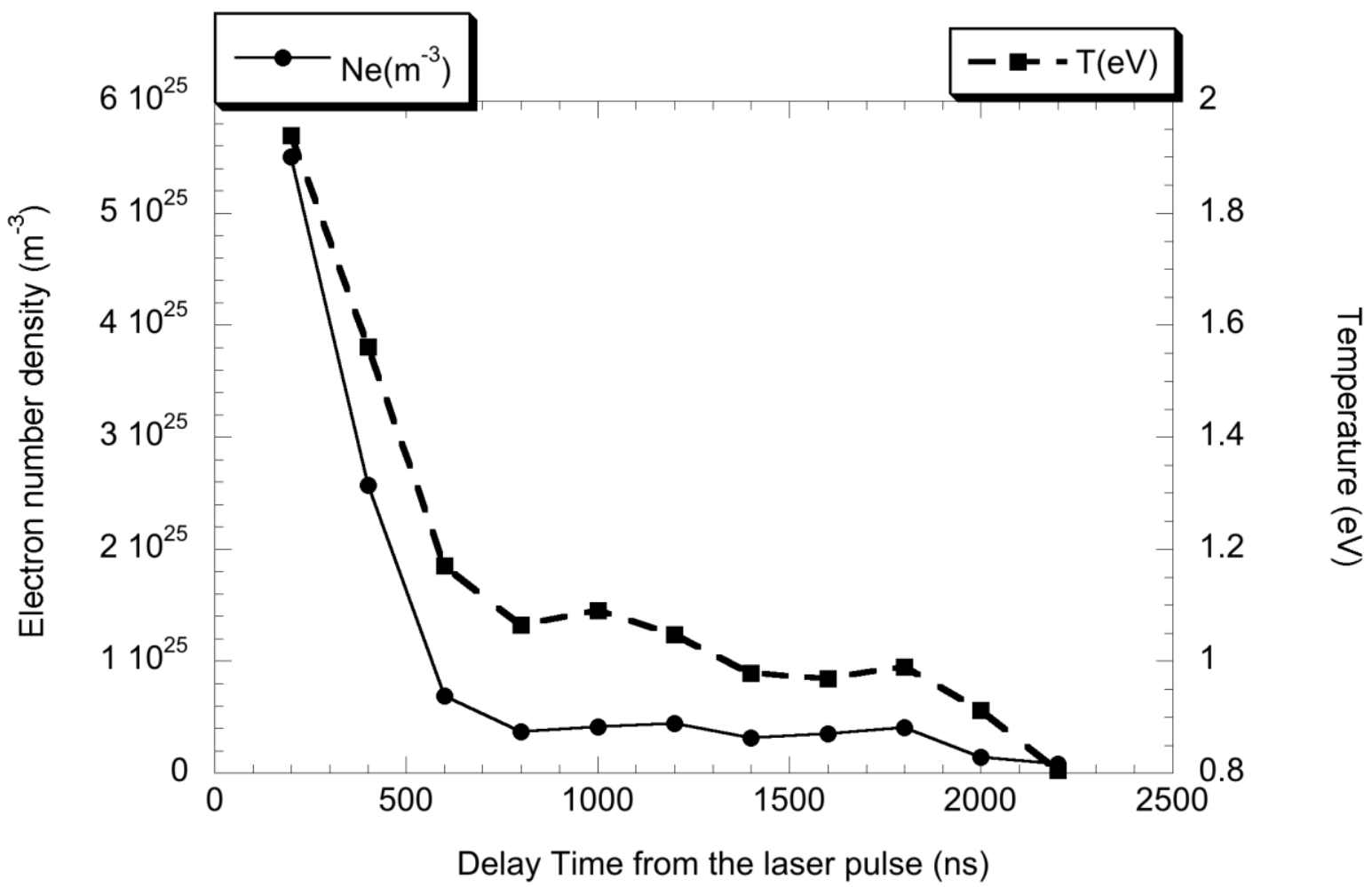

Fig.1. Plasma temperature (squares) and electron number density (circles) as function of time during LIBS on titanium target with laser irradiance $3 \mathrm{GW} \mathrm{cm}^{-2}$ at $1064 \mathrm{~nm}$.

By the inspection of Fig.1 it is observable that, in this experiment, during 1 microsecond after the laser pulse, the temperature decreases from $20000 \mathrm{~K}$ to $8000 \mathrm{~K}$ while the electron number density decreases of more than one order of magnitude. In order to classify the thermodynamic status of the LIP, as in the general for collisional plasma, the McWirther criterion can be applied to highlight the deviation from equilibrium [57-59]. McWirther criterion establishes a minimum value of electron number density for which collisional excitation/de-excitation process is at least 10 times faster than radiative processes and this can be expressed with the following formula:

$$
N_{e}>1.6 \cdot 10^{12} \sqrt{T_{e}} \cdot \Delta E_{u l}^{3}
$$

Eq. 5 shows that this critical number density depends by the square root of the temperature and by the cubic power of the energy jump of the considered transition. In particular it is important to underline that for assuming LTE condition it is important that the $\Delta \mathrm{E}_{\mathrm{ul}}$ refers to two adjacent levels with the maximum difference in energy of the entire electronic configuration of the considered species. In Fig.2 the electron number density evolution of the plasma obtained after the ablation of a ternary alloy $\mathrm{Fe} / \mathrm{Ni} / \mathrm{Co}$ is shown together with the critical electron number density value obtained with eq.5. It is visible that the experimental electron number density is always consistently higher than that one calculated by McWhirter criterion and this justify the assumption of LTE. 


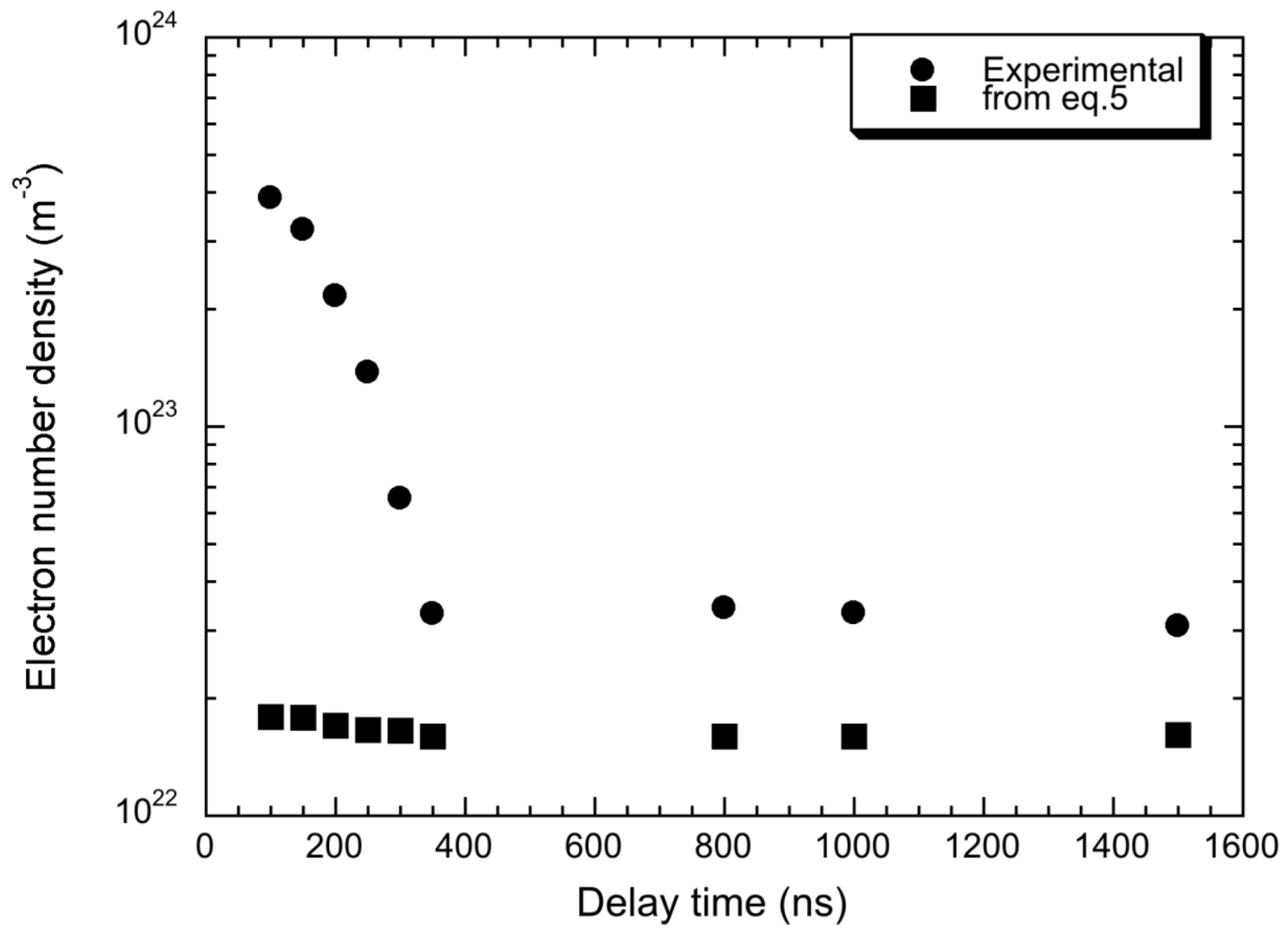

Fig.2. Comparison between the experimental electron number density during LIBS on Fe/Ni alloy [23] and the corresponding values obtained by eq.5.

If LTE is assumed it is possible to determine the composition of the plasma directly with plasma spectral emissions by introducing standard equilibrium relations in the general expression of emission intensity $[16,23,60]$ :

$$
I_{u l}=\frac{1}{4 \pi} A_{u l} h v_{u l} N_{u} G
$$

where $A_{\mathrm{ul}}$ is the Einstein coefficient, $v_{\mathrm{ul}}$ the transition frequency, $\mathrm{G}$ the instrumental factor and $\mathrm{N}_{\mathrm{u}}$ is the population in the upper level. If LTE is assumed $\mathrm{N}_{\mathrm{u}}$ is related to the total density of the species, $\mathrm{N}_{0}$, with the Boltzmann distribution:

$$
N_{u}=N_{0} \frac{g_{u}}{Z(T)} \exp \left(-\frac{E_{u}}{k T}\right)
$$


so that the following expression connecting the emission intensity to the total number density is obtained:

$$
N_{0, a}=\frac{I_{u l}}{4 \pi G h v_{u l} A_{u l} g_{u}} Z(T) \exp \left(\frac{E_{u}}{k T}\right)
$$

In eq. 8 the number density $\mathrm{N}_{0}$ is underestimated because the ionized fraction is neglected. For this reason we can calculate the amount of ionized species with the ionization equilibrium constant relation, namely Saha's equation:

$$
\frac{N_{0, i}}{N_{0, a}}=2 \frac{Z_{i}(T)}{Z_{a}(T)} N_{e}^{-1}\left(\frac{m_{e} k T}{2 \pi \hbar^{2}}\right)^{3 / 2} \exp \left(-\frac{E_{\text {Ion }}}{k T}\right)
$$

As a consequence of the decreasing of the electron number density during plasma evolution, the McWhirter criterion may be not satisfied for all the transitions. In Fig. 3 is shown an example with an Hydrogen laser induced plasma.

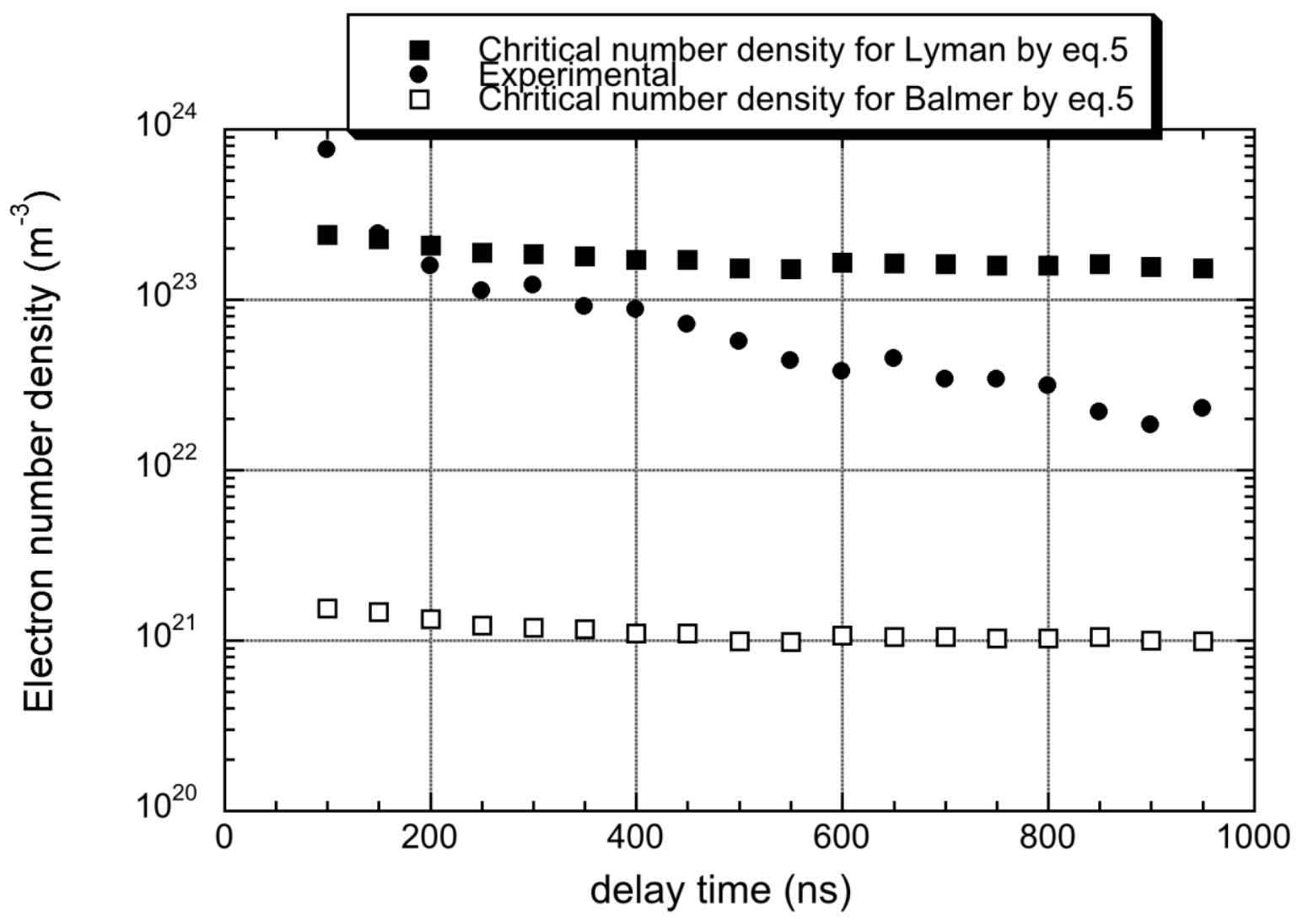


Fig.3. Comparison between the experimental electron number density of Hydrogen LIP at 100 Torr (circles) and the corresponding values obtained by eq.5 for the Balmer series (empty squares) and Lyman series (filled squares).

The Fig. 3 reports the experimental number density, the value of eq. 5 considering the $\Delta \mathrm{E}_{2-1}$ and the $\Delta \mathrm{E}_{3-2}$ representing respectively the main Lyman and Balmer transitions. It is interesting to note that by applying the McWhirter criterion, while Balmer's transition satisfies the eq. 5 for the investigated time interval and so is in a regime of collisional equilibrium, the Lyman's transition, that is characterized by an higher energy jump, is in equilibrium only in the first $200 \mathrm{~ns}$. After this time, as a consequence of the decrease of both electron number density and, in a smaller extent, of the excitation temperature, the rate of radiative processes of de-excitation becomes comparable with the rate of superelestic electron impact and LTE can not be assumed anymore. In other words the transitions involving the first two levels are out of the collisional equilibrium, while al the transitions connected to higher energy levels are still in a collisional regime. In this cases eedf Maxwell distribution and consequently heavy particles Boltzmann distribution do not hold anymore for the entire range of energy, but only for those energy levels whose transitions respect eq.5 and the plasma is in a partial Local Thermal Equilibrium (pLTE). When the LIP is in pLTE [52], equilibrium relation can not be applied and the plasma composition should be studied with state to state collisional radiative model with a non-linear system of coupled differential equations as following:

$$
\begin{aligned}
&\left(\frac{d N_{A^{Z}}(i)}{d t}\right)=-n_{e} N_{A^{z}}(i) \sum_{j} K_{i j}^{e x c}(n(\varepsilon))+n_{e} \sum_{j} K_{j i}^{\text {deexc }}(n(\varepsilon)) N_{A^{Z}}(j) \\
&-K_{i}^{i o n}(n(\varepsilon)) n_{e} N_{A^{Z}}(i)+K_{i}^{3 b}(n(\varepsilon)) n_{e}^{2} N_{A^{Z+1}}(0) \\
&-N_{A^{Z}}(i) \sum_{j<i} A_{i j}+\sum_{j>i} A_{j i} N_{A^{Z}}(j)+K_{i}^{R R}(n(\varepsilon)) n_{e} N_{A^{Z+1}}(0) \\
&\left(\frac{d N_{e}}{d t}\right)=\sum_{i}\left[K^{i o n}(n(\varepsilon)) n_{e} N_{A^{z}}(i)-K^{3 b}(n(\varepsilon)) n_{e}^{2} N_{A^{Z^{* 1}}}(0)\right]
\end{aligned}
$$

where $\mathrm{K}^{\text {exc }}, \mathrm{K}^{\text {deexc }}, \mathrm{K}^{\text {ion }}, \mathrm{K}^{3 \mathrm{~b}}$ and $\mathrm{K}^{\mathrm{RR}}$ represent the rate coefficients of direct and inverse processes in eqs.1-4, which depends on the eedf contained in the rate coefficient formulation. For a detailed description of this approach, interested reader can refer to Refs $[61,62]$.

\section{The recombining character of LIP and radiative emission.}

As briefly discussed in the previous sections, the LIP is a dynamic system where all the energy is delivered by the laser pulse and then the system evolves spontaneously for reaching the equilibrium with the surrounding environment. Initially the plasma is characterized by very high number density (close to the target density) and high temperature. In this view the plasma expands extremely fast 
decreasing the number density of ablated species of more than one order of magnitude. On the other hand also the temperature decreases shifting the balance of ionization reported in eq.3a in the backward direction. The overall effect is that the plasma, when the laser pulse is over, shows for the most of its persistence time a recombining character i.e. $\mathrm{dN}_{\mathrm{e}} / \mathrm{dt}<0[53,59]$. At typical laser plasma conditions the recombination time is longer than those associated to elastic collision between electrons and inelastic collision of excitation/de-excitation between electrons and heavy particles and then it does not perturb the Maxwell and the Boltzmann distributions of electrons and heavy particles. In any case the effect of recombination plays a crucial role in plasma emission and plasma duration. Two main effects can be addressed to the recombining character: the first is correlated to the average energy of the electrons $\varepsilon$ and the other one to the total energy of the electrons $E=n_{e} \varepsilon$. As a matter of fact the electrons that are involved in the recombination process are those with low energy. For this reason, when we deal with a recombining plasma, the eedf is depleted of the low energy fraction of electrons consequently increasing the fraction of high energy electrons and, in turn, the average energy of the distribution. This means that the plasma should increase the excitation temperature linearly as $\varepsilon$ $=3 / 2 \mathrm{kT}$.

On the other hand the number of total electron decreases as a consequence of the recombination with ions. The number of electrons that disappear because of the three body recombination is given by [53]

$$
\frac{d N_{e}}{d t}=-\alpha_{3 b} \cdot N_{e}^{3}
$$

where $\alpha_{3 b}$ is the rate coefficient of the three body recombination process neglecting multiple step of ionization.

$$
\alpha_{3 b}=10^{-14} \cdot \frac{\sigma_{0}}{E_{\text {ion }}} \cdot\left(\frac{T}{E_{\text {ion }}}\right)^{-\frac{9}{2}}
$$

In eq.12 $\alpha_{3 \mathrm{~b}}$ is given in $\mathrm{cm}^{6} \mathrm{~s}^{-1}, \mathrm{~T}$ and the ionization energy $\mathrm{E}_{\text {ion }}$ in $\mathrm{eV}$, while $\sigma_{0}$ is the geometrical atomic cross section [63]. By eqs. 11-12 it is clear that while $\varepsilon$ depends linearly on T, the decrease of electron number density depends on $\mathrm{T}^{-9 / 2}$. In this connection, when $\mathrm{T}$ changes, the increase of $\varepsilon$ do not compensate the faster disappearing of electrons with the net result that the total energy of electrons decreases and the eedf immediately rearrange to a lower temperature because the elastic impacts are very fast. Being the heavy particles in equilibrium with electrons, the net decreasing of the electrons energy is compensated by the heavy particles with spontaneous emission. In other words the disappearing of slow electrons, implies the overpopulation of high excited states of atoms and the consequent radiative decay. In this frame there is direct correlation between the number of electrons disappeared because of recombination and the emission intensity of atoms that can be written as [46, 55]: 


$$
\frac{4 \pi I_{u l}}{h v_{u l} G}=\left|\Delta N_{e}\right| N_{l} k_{l u}+A_{u l} N_{u}
$$

where $\Delta \mathrm{N}_{\mathrm{e}}$ is the number of electron disappeared because of recombination, $\mathrm{k}_{\mathrm{lu}}$ is the rate of deexcitation. The second term in eq. 13 take into account of standard spontaneous emission contribution. In eq. 13 it can be noted that when the recombination proceed very efficiently the main contribution to the emission is given by the recombination, while at late delay, when temperature and electron number density stabilize, the second term become predominant.

\section{High Density effect}

The initial stage of LIP evolution is characterized by continuum radiation due to the processes involving free electrons [64]. Immediately after the laser pulse the plasma is almost completely ionized with a degree of ionization close to unity and the density of the plasma is close to that one of the irradiated target. At this condition high density effects should be considered for a correct interpretation of the plasma features. Being the temperature sufficiently high (tens of thousands of Kelvin) the plasma can still be treated like an ideal plasma obeying to Maxwell-Boltzmann statistics $[64,65]$, but the effect of the electric fields of charged particles results in a dramatic decrease of the ionization energy of atoms and ions, in agreement with the Debye-Hückel theory $[46,66]$. The decrease of ionization energy is given by [67]:

$$
\begin{aligned}
& \mathrm{E}_{\text {ion }}^{\prime}=\mathrm{E}_{\text {ion }}-\Delta \mathrm{I} \\
& \Delta I=\frac{e_{0}^{2}\left(z_{s}+1\right)}{4 \pi \varepsilon_{0} \lambda_{D}}
\end{aligned}
$$

where $\lambda_{D}$ is the Debye radius and $\mathrm{z}$ the ionic formal charge, $\mathrm{e}_{0}$ is the electron charge and $\varepsilon_{0}$ is electric permittivity of vacuum. It is important to note that eq. 14 is directly obtained from thermodynamic considerations and so it is adequate for non-hydrogenoid species too. Due to the lowering of ionization energy, the highest bound energy level available for emitters corresponds to the difference between the ionization energy of isolated species and $\Delta \mathrm{I}$ as reported in Fig.4. 


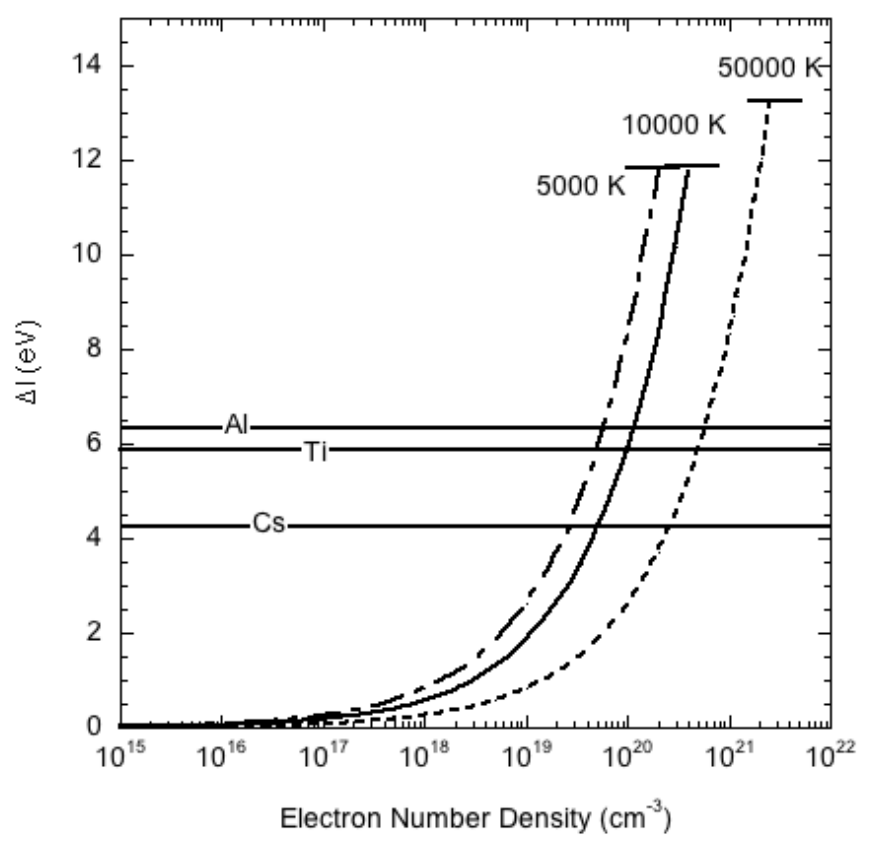

Fig.4. Effect of the electron number density on the decreasing of ionization energy calculated by eq.14b.

For this reasons the number of allowed excited levels are strongly limited with the net result that excitation/de-excitation processes are replaced by ionization and radiative recombination. For this reason discrete emission lines are detectable only after a certain delay time from the laser pulse when, as a consequence of the expansion and recombination, the electron number density decreases from $10^{22} \mathrm{~cm}^{-3}$, immediately after the breakdown, to $10^{19} \mathrm{~cm}^{-3}$. Fig.5 show the temporal evolution of continuum radiation, ionic lines and atomic lines [64]. By the observation of Fig.5 it is possible to see that initially there is only continuum radiation, then ionic lines appear for first, because they have higher ionization energy $E_{\text {ion }}$ (see eq.14a) than atomic ones, and finally also atomic lines take place. 


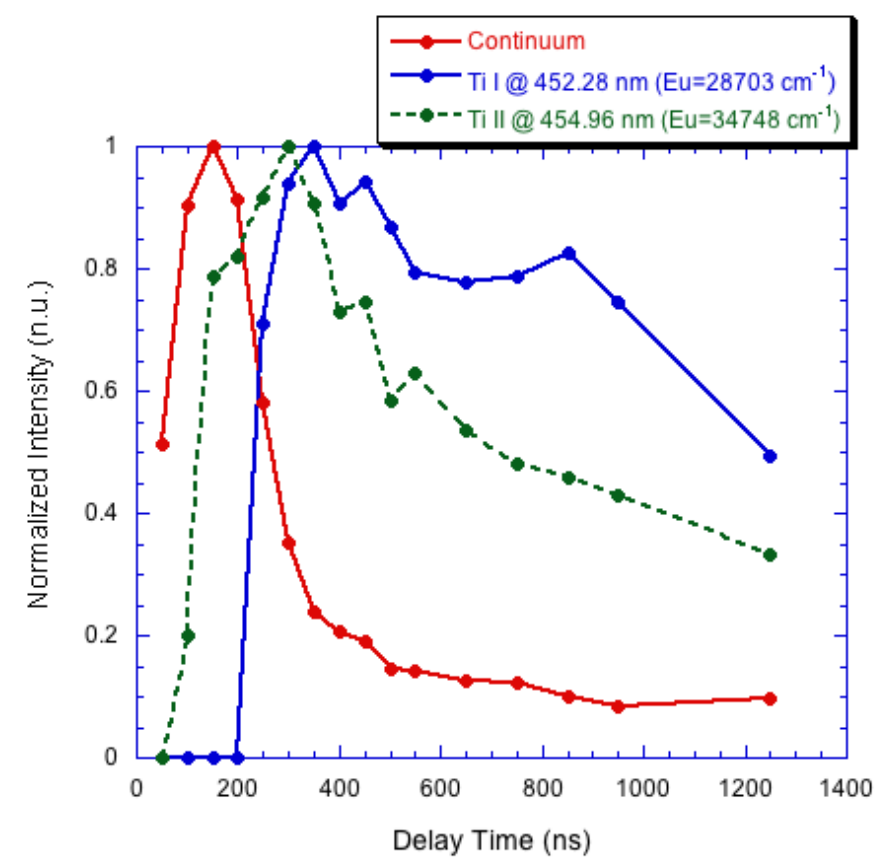

Fig.5. Temporal evolution of continuum radiation, atomic emission and ionic emission from Ref. [64].

In general continuum radiation is due to two processes: free-free transition (Bremsstrahlung) and freebound transition (radiative recombination) according to the following equation [8]:

$$
\varepsilon_{c}=\left\lfloor\frac{16 \pi e^{6}}{3 c^{3}\left(6 \pi m^{3} k\right)^{1 / 2}}\right\rfloor \frac{N_{e}^{2}}{T^{1 / 2}}\left\lfloor\xi_{f-b}\left(1-\exp -\frac{h v}{k T_{e}}\right)+G_{f-f} \exp -\frac{h v}{k T_{e}}\right\rfloor
$$

where $\xi_{\mathrm{f}-\mathrm{b}}$ and $\mathrm{G}_{\mathrm{fff}}$ are respectively the Gaunt factor for free-bound and free-free transitions. The freefree transition are efficient in the very beginning of plasma evolution (generally a few tens of nanoseconds after the laser pulse) when the temperature is very high and the Debye Huckel effect is so strong that heavy particles behave just like elementary particles. On the contrary for the most of the time that the continuum radiation is detectable, it is the results of free-bound transition, i.e. radiative recombination.

\section{Atomic emission in LIP.}




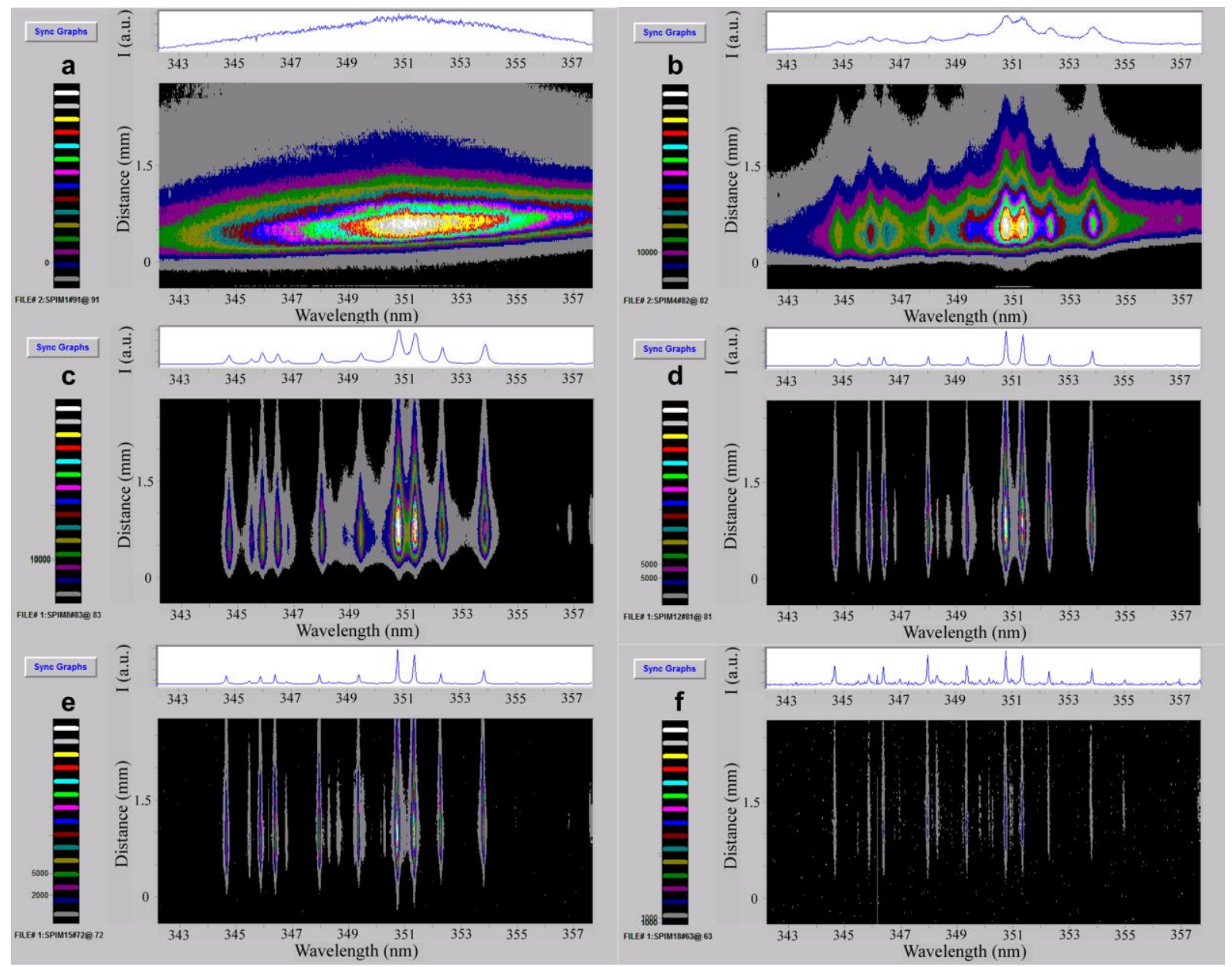

Fig.6. Spectral resolved images during LIBS on Titanium at different delay time from the laser pulse: a) $50 \mathrm{~ns}$; b) $200 \mathrm{~ns}$; c) $400 \mathrm{~ns}$; d) $650 \mathrm{~ns}$; e) $950 \mathrm{~ns}$; f) $3250 \mathrm{~ns}$. Gate width $50 \mathrm{~ns}$.

In Fig.6, spectrally resolved images of Ti-LIP are shown for different delay times from the laser pulse. As a first observation on the ordinate is reported the distance from the target along the propagation axis of the plasma and it is shown that after $50 \mathrm{~ns}$ the plasma center of mass reaches $0.5 \mathrm{~mm}$ while after $3.2 \mu \mathrm{s}$ it reaches $1.5 \mathrm{~mm}$ and so it pass from an initial velocity around $10^{6} \mathrm{~cm} \mathrm{~s}^{-1}$ to $410^{5} \mathrm{~cm} \mathrm{~s}^{-1}$ after a few microseconds. As a consequence of the expansion the plasma temperature decreases as discussed above and the hierarchy of the elementary mechanisms changes in agreement with the previous discussion. It is possible to see the effect of the elementary mechanisms competition on the emission spectra. At very short delay the high density effects limit the number of accessible levels and the emission is dominated by a strong continuum radiation (Fig.5a). As a consequence of the plasma expansion and recombination the electron number density and the total density decrease and emission lines start to rise up from the continuum radiation spectrum. As the electron number densities is very high $\left(>10^{19} \mathrm{~cm}^{-3}\right)$ only emission lines corresponding to ions or to atomic transition with low energy excited level are detectable. All the emission lines appear broad as a consequence of the Stark broadening and of self-absorption. As the expansion and recombination proceed, more and more atomic lines are visible. Moreover as the Stark broadening depends mainly by the electron number density [8] the line profile becomes narrower with time. It is possible to see that from $400 \mathrm{~ns}$ of delay from the laser pulse the emission lines shows the excited level population in a Boltzmann form in agreement with what has been discussed about the LTE conditions. Indeed the electron number 
density, estimated from the Stark broadening in agreement with the data reported in Ref. [5], varies from $710^{18} \mathrm{~cm}^{-3}$ at $400 \mathrm{~ns}$ to $210^{17} \mathrm{~cm}^{-3}$ at $3.2 \mu$ s, holding eq.5 valid in the case of Ti in this range of time.

Until now the fundamental aspects of the atomic plasma produced by laser matter interaction have been discussed considering the plasma homogenous and optically thin [8]. In the real experiments these condition can fail introducing several question on the trueness of the LTE plasma equations [59, $68]$.

As an example, as a consequence of the plasma expansion and its interaction with the surrounding environment, the excitation temperature is different in different zones of the plasma. In this case the temperature in the core of the plasma can be much higher than that one in the border of the plasma. This issue has been investigated with Abel inversion by several authors [69-71]. For example Parigger at. al [70] have found that the electron density distributions reveal spherical shell structures and indicate a change in temperature of a factor of 2 across the radial dimension. In any case this kind of approach should be taken with caution and supported by theoretical issue [72].

Another important question in studying the emission of laser induced plasma is the self absorption. Self-absorption occurs to various extents when the plasma can not be considered optically thin and the emitted radiation from atoms, ions and molecules in the plasma is absorbed along the optical path by the same species in lower levels [71]. Self absorption can manifest in two ways. Absorption can occur because the species in the plasma at the same temperature and density absorb the emitted radiation [73]. In this case the line intensity profile appears corrupted but still symmetric. This kind of effect regards high concentration species and transition involving low energy levels. Another self-absorption effect is when colder section of the plasma absorbs the emission coming from the hotter core $[50,68,74]$. This generally (but not necessarily) occurs at long delay from the laser pulse, and give selfinversed lines and asymmetrical absorption. Several methods have been proposed to take into account of self absorption that as a matter of fact gives an underestimation of the emitted intensity and in turn of the population of the species in the considered excited level. By the experimental point of view Moon et al. [75] proposed the double mirror technique in order to estimate the self-absorption percentage and determine a correction factor for each line. This method allows to strongly improve the sensitivity of calibration curve in elemental analysis application.

As a consequence of the explosive nature of the laser ablation, the plasma can have a different spatial distribution of the species. Aragon et. al [76] have shown that during the ablation of a Fe-Si target, the distributions of the emission coefficient of $\mathrm{Fe}, \mathrm{Si}$ and $\mathrm{H}$ lines show that they are emitted from different regions of the plasma and this can affect the trueness of the traditional space integrated measurements.

Finally also in the best of the case, the LTE can hold since the electron number density decreases beyond the McWhirter critical value and then new processes involving heavy particles collisions should be considered. In this view in the next sections the formation of molecules and their emission features will be discussed.

\section{Plasma chemistry: equilibrium approach versus kinetic modeling}

The theoretical prediction of molecule formation in laser plasmas by numerical modeling has been proposed using different approaches. Itina et al performed Monte Carlo simulations to describe nonequilibrium chemical reactions during pulsed laser deposition under vacuum [77]. Their investigations suggested a strong influence of chemical reactions on the angular distribution of species within the ablation plume. Using a one-dimensional fluid model, combined with the assumption of local 
thermodynamic equilibrium (LTE), Colonna and coworkers predicted the formation of TiO molecules during ablation of titanium monoxide for thin films synthesis [78]. Hermann and Dutouquet performed LTE modeling to predict the formation of oxides and nitrides from ablation of aluminum, titanium and graphite in low-pressure oxide or nitrogen atmospheres [79]. Using a hybrid model that combines collective and diffusive processes, Itina et al predicted the localized formation of AlO radicals in the volume of vapor-gas mixing [80]. Babushok et al [81] developed a kinetic model to describe the reaction kinetics during LIBS analysis of cyclotrimethylenetrinitramine (RDX). The authors predicted a process of two successive stages: (i) the fast establishment of a quasi-stationary state after laser excitation, and (ii) the slow evolution of the quasi-stationary state according to the plasma temperature evolution. They concluded that the initial mechanisms of RDX decomposition have negligible influence on the observable LIBS signature. Shabanov and Gornushkin combined LTE modeling with a fluid dynamic model to evaluate the role of chemical reactions [82]. Their analyses indicate that chemical reactions occur mostly in the plasma periphery where the temperature is low and vapor-gas intermixing takes place. The same conclusion was made by Hermann et al for ablation of aluminum in air when comparing measured atomic and molecular emission to the spectral radiance of a nonuniform reactive LTE plasma [50]. The simulations show that atomic lines are mainly emitted from the hot plasma core while molecular band emission originates from the cold plasma periphery, where the atomic emission is reabsorbed.

The different approaches of plasma chemistry modeling illustrate two main tendencies: the equilibrium approach and the kinetic approach. Both approaches naturally apply to different experimental conditions. The equilibrium case is more appropriate to describe plasmas in typical LIBS conditions, produced by nanosecond lasers in air or inert gas at atmospheric pressure [50, 82]. In a low pressure gas environment, or under short pulse laser excitation, the equilibrium conditions are not fulfilled and a kinetic approach is required [77, 81]. Only non-equilibrium plasma kinetics is expected to enable a correlation between native bonds in organic materials and LIBS emission spectra. Here, femtosecond laser beam excitation seems to be more appropriate to obtain information about the molecular structure within the material from the LIBS signal [43, 83]. Serrano et al evidenced the correlation for deuterated molecular solids [84]. The isotopic labeling allowed the authors to identify the origin of the diatomic species from which the emission bands were observed. In addition, Serrano et al evidenced the competition of atmospheric oxygen with nitrogen in the reactions. In a former work, isotopic labeling was used by Dong and coworkers to study chemical processes in traditional LIBS conditions, using nanosecond infrared laser radiation [85]. The authors concluded that $\mathrm{C}_{2}$ radicals are mainly produced by recombination processes, excluding thus the direct release of $\mathrm{C}_{2}$ from the sample. Negre et al performed time- and space-resolved analysis of molecular and atomic emission during nanosecond laser irradiation of various polymer materials [86]. The authors were able to correlate the observed emission dynamics to the presence of single and double bonds in the molecular solid. However, the presented results do not directly prove for the contribution of native bonds to the observed plasma emission. The tribological properties (i.e. hardness) combined with the elemental composition of the polymer samples may also explain the observed emission features.

Table 1. Energies $T_{u}$ and $T_{l}$ of upper and lower electronic states, respectively, of the most prominent molecular systems. The first excited electronic state of the molecule contributes significantly to the partition function if the corresponding energy $T_{n=2}$ is small. The CO Angström system was observed in non-equilibrium plasmas only.

\begin{tabular}{cccccc}
\hline molecule & system & transition & $T_{u}(\mathrm{eV})$ & $T_{l}(\mathrm{eV})$ & $T_{n=2}(\mathrm{eV})$ \\
\hline $\mathrm{CN}$ & violet & $B^{2} \Sigma^{+}-X^{2} \Sigma^{+}$ & 3.19 & 0 & 1.14 \\
$\mathrm{C}_{2}$ & swan & $d^{3} \Pi_{\mathrm{g}}-a^{3} \Pi_{\mathrm{u}}$ & 2.48 & 0.09 & 0.09 \\
$\mathrm{AlO}$ & blue-green & $B^{2} \Sigma^{+}-X^{2} \Sigma^{+}$ & 2.56 & 0 & 0.66 \\
\hline
\end{tabular}




\begin{tabular}{cccccc}
\hline $\mathrm{TiO}$ & gamma & $A^{3} \Phi-\mathrm{X}^{3} \Delta$ & 1.76 & 0 & 0.43 \\
$\mathrm{~N}_{2}{ }^{+}$ & first negative & $B^{2} \Sigma_{\mathrm{u}^{+}}-X^{2} \Sigma_{\mathrm{g}}{ }^{+}$ & 3.16 & 0 & 3.16 \\
$\mathrm{CO}$ & Angström & $B^{1} \Sigma^{+}-A^{1} \Pi$ & 10.78 & 8.07 & 6.04 \\
\hline
\end{tabular}

\section{Diagnostics using molecular emission}

The above mentioned classification in equilibrium and non-equilibrium plasma chemistry and the related question on the possible correlation between native bonds and observed spectral emission is still an open topic and more experimental data are required to validate the appropriate model in particular experimental conditions. In this context, accurate plasma diagnostics are of interest and several authors report on temperature measurements based on the analysis of molecular emission bands. Lam et al measured the rovibrational temperature of AlO during laser ablation of Ti-doped aluminum oxide. To minimize the measurement error due to self-absorption, the $(0,0)$ band head of the blue-green system (see table 1) was excluded from the molecular spectra analysis. Comparing the rovibrational temperature to the electronic excitation temperature of $\mathrm{Ti}$ ions, the authors found a significant difference and concluded that the plasma was out of equilibrium. They ignored however the temperature gradient that may explain the preferential emission of molecules and ions from the cold periphery and the hot plasma core, respectively [50, 82]. Although mostly ignored in molecular spectra analysis, self-absorption is expected to alter the intensity distribution of band emission as the observed molecular systems generally occur between electronic levels of low excitation energy (see table 1). For example, strong absorption of the $\mathrm{C}_{2} \mathrm{~S}$ wan system was evidenced by Vivien et al during graphite ablation in low pressure nitrogen [87]. Self-absorption predominantly affects the rotational lines of largest line strength and lowest excitation energy. Consequently the change of the rovibrational intensity distribution may lead to an overestimation of the deduced temperature, if selfabsorption is ignored [50]. This may explain the observed increase of the TiO rovibrational temperature observed in Ref.[46] for late times.

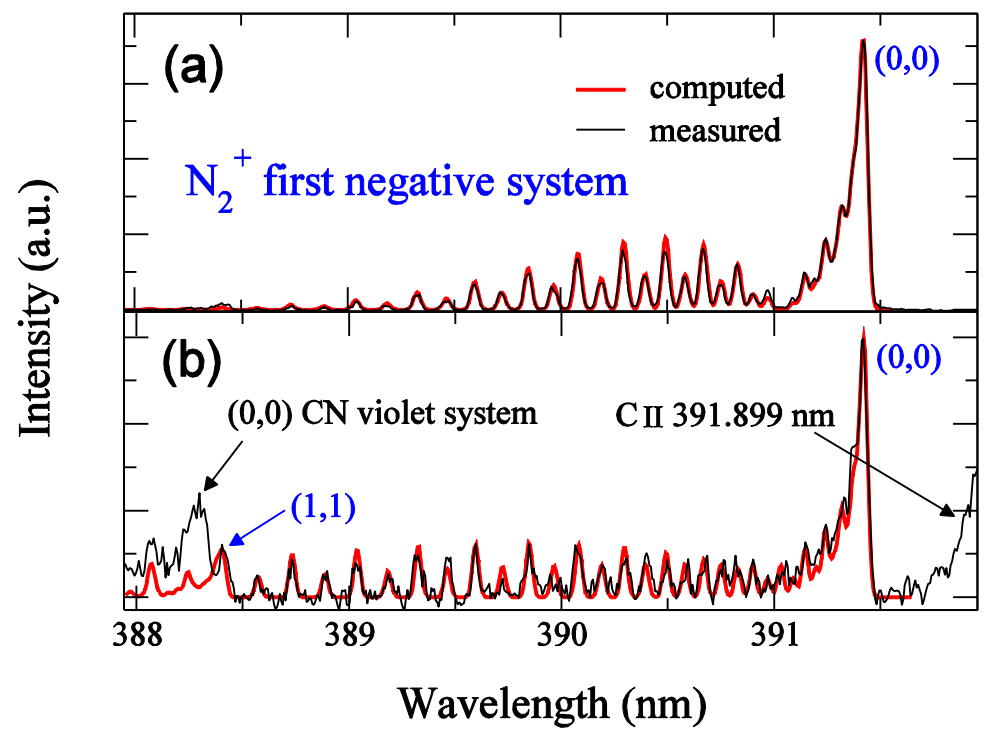

Fig. 7. The $(0,0)$ band of the $\mathrm{N}_{2}{ }^{+}$first negative system recorded during nanosecond laser ablation in nitrogen at $130 \mathrm{~Pa}$ pressure for $t=40 \mathrm{~ns}$ (a) and $t=170 \mathrm{~ns}$ (a). The computed spectra were obtained for rotational temperatures of $320 \mathrm{~K}$ (a) and $1200 \mathrm{~K}$ (b) [87]. 
A particular non-equilibrium situation is illustrated in fig 7 where the $(0,0)$ band of the first negative system of $\mathrm{N}_{2}{ }^{+}$is displayed for laser ablation of graphite in low pressure nitrogen [87]. The spatially resolved measurements correspond to a distance of $2 \mathrm{~mm}$ from the sample surface and two different delays. The spectrum recorded for $t=40 \mathrm{~ns}$ (a) corresponds to the time when the shock wave reaches the observed volume. The deduced rotational temperature equals the room temperature. The low temperature is in complete opposition to the energy as large as $19 \mathrm{eV}$ required for ionization and excitation of molecular nitrogen. This reveals the strong coupling of free electrons with the electronic states, and their weak coupling with the vibrational and rotational motions. Later, at $t=170 \mathrm{~ns}(\mathrm{~b})$, the ablated vapor species arrive in the observation volume, and the rotational temperature of $\mathrm{N}_{2}{ }^{+}$increases due to collisions with heavy particles. However, the situation illustrated by fig. 7 is characteristic for ablation in low pressure atmospheres exclusively. In typical LIBS plasmas, generated at atmospheric pressure, thermalization occurs on a much faster timescale due to the large collision rates at high density. Consequently, the rotational and vibrational temperatures deduced from molecular emission bands generally do not differ within the measurement uncertainty [50, 88, 89]. We stress that the "beautiful aspect" observed in many molecular spectra simulations is not necessarily associated to an accurate temperature measurement: the small energy gap between the rotational lines and/or vibrational bands combined with the low accuracy of the available spectroscopic data, and possible self-absorption may lead to large measurement errors. Similar to the error evaluation of the atomic excitation temperature, the uncertainty of the temperature deduced from molecular spectra can be estimated from [16]

$$
\frac{\Delta T}{T}=\frac{k T}{\Delta E} \frac{\Delta R}{R}
$$

where $\Delta E$ stands for the maximum energy gap of involved rotational and/or vibrational levels in the upper electronic state, $R$ is the corresponding intensity ratio. Beside measurement errors due to the apparatus response or to noise, the uncertainty $\Delta R$ also accounts for intensity calculation errors associated to the uncertainty of molecular constants.

\subsection{Formation and observation of molecular species}

Table 2. Classification of diatomic molecules and radicals into three categories according to their dissociation energy from the ground state $D_{0}:$ large $\left(D_{0}>6 \mathrm{eV}\right)$, moderate $\left(3 \mathrm{eV}<D_{0}<6 \mathrm{eV}\right)$ and small dissociation energy $\left(D_{0}<3 \mathrm{eV}\right)$. For each species $D_{0}$ is given in $\mathrm{eV}$ in the parentheses [90].

\begin{tabular}{|c|c|c|}
\hline category & $D_{0}$ & molecule or radical \\
\hline A & large & $\begin{array}{c}\mathrm{CO}(11.1), \mathrm{N}_{2}(9.8), \mathrm{BO}(8.3), \mathrm{SiO}(8.3), \mathrm{HfO} \\
(8.2), \mathrm{CN}(7.8), \mathrm{ZrO}(7.8), \mathrm{YO}(7.4), \mathrm{TiO}(6.9) \\
\mathrm{AlF}(6.9), \mathrm{NO}(6.5), \mathrm{C}_{2}(6.2)\end{array}$ \\
\hline B & moderate & $\begin{array}{c}\mathrm{BaO} \text { (5.8), } \mathrm{CaF}(5.5), \mathrm{AlO}(5.3), \mathrm{O}_{2}(5.12), \mathrm{SrO} \\
\text { (4.9), } \mathrm{TiN}(4.9), \mathrm{H}_{2}(4.8), \mathrm{CaO}(4.7), \mathrm{BC}(4.6), \mathrm{OH} \\
\text { (4.4), } \mathrm{CaCl}(4.3), \mathrm{HCl}(4.3), \mathrm{FeO}(4.2), \mathrm{NaCl}(4.2), \\
\mathrm{BN}(4.0), \mathrm{CH}(3.5)\end{array}$ \\
\hline $\mathrm{C}$ & small & $\begin{array}{c}\mathrm{CuO} \text { (2.8), } \mathrm{AuO}(2.3), \mathrm{Cu}_{2}(2.0), \mathrm{Al}_{2}(1.9), \mathrm{Ti}_{2} \\
(1.3), \mathrm{Fe}_{2}(1.1)\end{array}$ \\
\hline
\end{tabular}


The presence of molecular species in the plasma critically depends on the dissociation energy $D_{0}$ that is the minimum energy to be delivered to separate both atoms from the ground state of the molecule. In table 2, the most prominent diatomic species are presented and classified into three categories according to their $D_{0}$-values. The most stable molecules (category A) have dissociation energies as large as or even larger than the first ionization energy of many metals. Accordingly, these molecules can exist in strongly ionized plasmas, as demonstrated by numerous observations of $\mathrm{CN}, \mathrm{C}_{2}$ during the early stage of laser-produced plasmas, when the electron density is high [25, 86, 87, 91, 92]. The category $\mathrm{B}$ composes molecules with intermediary $D_{0}$ that are observable at sufficiently low temperature, typically in the cold plasma periphery or during the late stage of plasma expansion [48, 50]. The molecules of low $D_{0}$ (category $C$ ) are hardly observable in equilibrium plasmas as they are formed at temperatures too low for their electronic excitation.

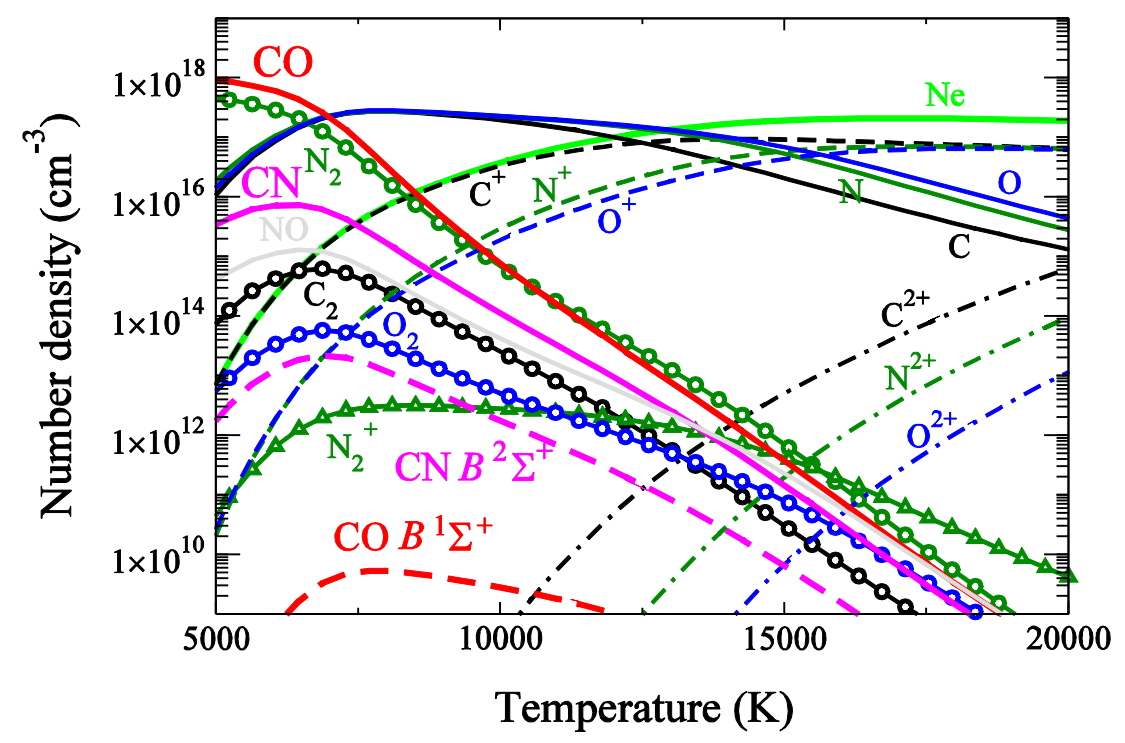

Fig. 8. Number densities of species vs temperature computed for a plasma in local thermodynamic equilibrium at atmospheric pressure composed of carbon, nitrogen and oxygen of equal atomic fraction (see calculation details in section 8).

A particular case is the $\mathrm{CO}$ radical: although it presents the largest dissociation energy, $\mathrm{CO}$ is not observed in collisionally dominated LIBS plasmas. According to the large upper level energies of the CO Angström system (see table 1) and the other electronic transitions [28], efficient excitation can only occur at high plasma temperature, when the radical is mostly dissociated. This is illustrated in fig. 8 where the composition of a plasma composed of carbon, oxygen and nitrogen is presented as a function of temperature. The number densities of species were calculated for atmospheric pressure assuming local thermodynamic equilibrium. In addition to atomic and molecular species, the population number densities of the upper electronic states of the $\mathrm{CN}$ violet and $\mathrm{CO}$ Angström systems are presented. It is shown that, unless the large number density of $\mathrm{CO}$ in the temperature range $<7000$ $\mathrm{K}$, the $\mathrm{CO} B{ }^{1} \Sigma^{+}$state has a very low population number density that explains the absence of $\mathrm{CO}$ emission in LIBS experiments. Compared to the $\mathrm{CO} B{ }^{1} \Sigma^{+}$state, the upper state of the CN violet system reaches an about $10^{4}$ times larger population number density, although the $\mathrm{CN}$ number density is about 2 orders of magnitude smaller than the $\mathrm{CO}$ number density. The strong emission of $\mathrm{CN}$ and the absence of $\mathrm{CO}$ emission bands are thus expected for plasmas from vaporized organic materials close to equilibrium. Out of equilibrium, the emission bands of the CO Angström system are currently 
observed [93-95], and the absence of CO emission in LIBS plasmas indicate a plasma state close to equilibrium.

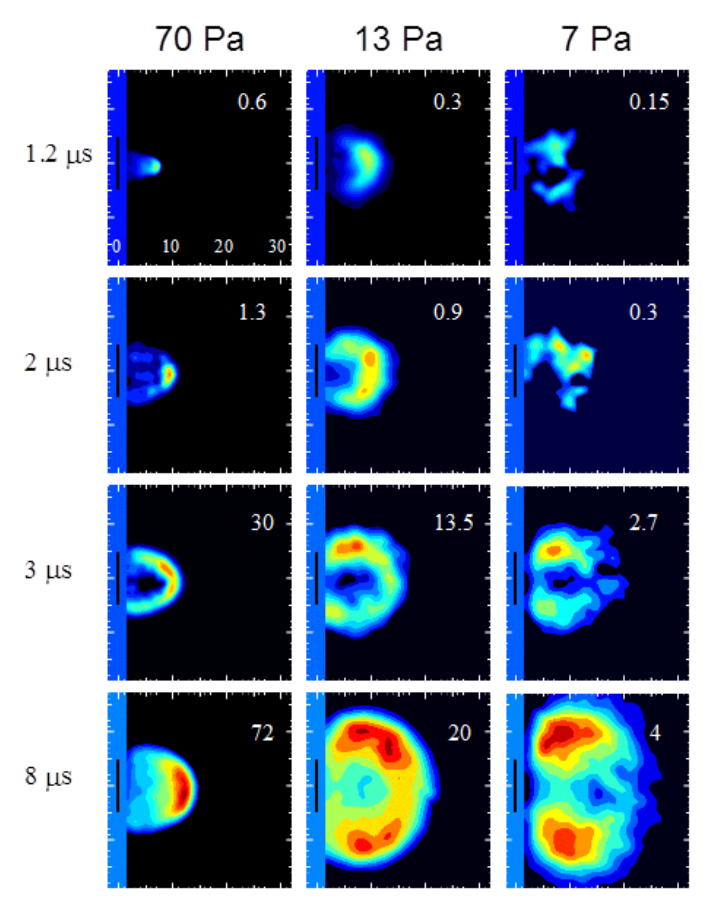

Fig. 9. Spatial distribution of $\mathrm{AlO}$ ground state population number densities obtained by laser-induced fluorescence probing for different times and oxygen pressures. The maximum number density is given on each image in $10^{13} \mathrm{~cm}^{-3}[96]$.

In summary, the observation of molecular emission bands requires three conditions: (i) the temperature is appropriate (low enough to enable the molecular formation and high enough for the electronic excitation), (ii) the atomic reactants are present with sufficiently large density (intermixing between ablated vapor and ambient gas in case of vapor-gas chemical reactions) and, (iii) the molecular transition is observable (upper level energy low enough to enable the electronic excitation). When the plasma temperature is too low for the electronic excitation, laser-induced fluorescence (LIF) may be applied to probe molecular species. This is illustrated in fig.9, where the two-dimensional distribution of $\mathrm{AlO}$ ground state radicals is presented for laser ablation of aluminum in low pressure oxygen atmospheres. The LIF images recorded for different delays with respect to the ablating laser pulse were obtained by transforming the probe laser beam in a thin plane section. The maximum number density given on each image in $10^{13} \mathrm{~cm}^{-3}$ was obtained by calibration with absorption measurements. The images show the preferential formation of $\mathrm{AlO}$ in the volume of strongest intermixing, resulting from a vortex movement that was predicted theoretically [80].

\section{Calculation of the equilibrium plasma composition}

In the non-equilibrium case, the chemical pathway is of primary interest and all significant reactions have to be considered to predict the plasma chemistry by a kinetic model [81]. Contrarily, in the equilibrium case, all physico-chemical processes are counterbalanced and the presence of atomic and molecular species is determined solely by their formation energies and partition functions. The 
statistical laws of equilibrium describe the plasma state, ignoring the individual processes. The composition of a plasma composed of $M$ elements depends on $M+1$ parameters: the temperature and the atomic number densities of the $M$ elements. In the temperature range of interest the formation of polyatomic molecules can be neglected and the atomic number density of an element $A$ is given by

$$
n_{A}=\sum_{z=0}^{z_{\max }} n_{A}^{z}+2 \sum_{z=0}^{1} n_{A_{2}}^{z}+\sum_{B \neq A} \sum_{z=0}^{1} n_{A B}^{z}
$$

Here, $n_{A}^{z}$ and $n_{A_{2}}^{z}$ are the number densities of atomic and homonuclear diatomic species of charge $z$, respectively, and $n_{A B}^{z}$ are the number densities of heteronuclear diatomic species of charge $z$ formed by chemical reactions with the element $B$. The sum includes all species of significant abundance up to the maximum charge $z_{\max }$. The number densities of diatomic molecules formed by chemical reactions between the elements $A$ and $B$ were obtained from the Guldberg-Waage law of mass action for chemical equilibrium [97]

$$
\frac{n_{A}^{0} n_{B}^{0}}{n_{A B}^{0}}=\frac{(2 \pi \mu k T)^{3 / 2}}{h^{3}} \frac{Q_{A}^{0} Q_{B}^{0}}{Q_{A B}^{0}} e^{-D_{0} / k T},
$$

where $n_{A}^{0}$ and $n_{B}^{0}$ are the number densities of neutral atoms of elements $A$ and $B$, respectively, and $n_{A B}^{0}$ is the density of diatomic molecules formed by chemical reactions between both elements. $Q_{A}^{0}$, $Q_{B}^{0}$, and $Q_{A B}^{0}$ are the corresponding partition functions and $\mu=m_{A} m_{B} /\left(m_{A}+m_{B}\right)$ is the reduced mass. Thus, for given values of temperature and atomic number densities $n_{A}$ of the $M$ elements, the number densities of all plasma species can be calculated by numerically solving the equations that govern the LTE plasma [79]. Once the number densities of all species are calculated, the electron density is obtained from the equation of charge neutrality by summing the densities of all charged particles,

$$
n_{e}=\sum_{A} \sum_{z=1}^{z_{\max }} z n_{A}^{z}
$$

For practical applications, the atomic number densities $n_{A}$ [see eq.17] of the $M$ elements can be replaced by an equivalent set of input parameters: the electron density and the atomic or mass fractions of $M-1$ elements. The atomic fraction of each element $C_{A}$ is obtained by dividing $n_{A}$ by the sum over the atomic number densities of all elements $n_{\text {tot }}=\sum_{A} n_{A}$ :

$$
C_{A}=n_{A} / n_{\text {tot }}
$$

The number densities of plasma species computed for local thermodynamic equilibrium displayed in fig. 8 were obtained by setting the kinetic pressure of the plasma

$$
P=\left[n_{e}+\sum_{A} \sum_{z=0}^{z_{\max }}\left(n_{A}^{z}+n_{A_{2}}^{z}+\sum_{B \neq A} n_{A B}^{z}\right)\right] k T-\Delta P_{D}
$$

to atmospheric pressure. In eq. $21, \Delta P_{D}$ is the Debye pressure correction that accounts for the potential energy of charged species in the plasma. 


\section{Calculation of molecular emission}

In the simplest case of a uniform plasma in local thermodynamic equilibrium, the spectral radiance is given by [98]

$$
B_{\lambda}=U_{\lambda}\left(1-e^{-\tau}\right)
$$

where $U_{\lambda}$ is the blackbody spectral radiance and $\tau$ the optical thickness that characterizes the transmittance of the optical medium. The optical thickness is given by $\tau=\int \alpha(z) d z=\alpha L$, where $L$ is the diameter of the plasma along the observation direction and $\alpha$ the absorption coefficient given by [8]

$$
\alpha(\lambda)=\pi r_{0} \lambda^{2} f_{l u} n_{l} P(\lambda)\left(1-e^{-h c / \lambda k T}\right) .
$$

Here, $r_{0}$ is the classical electron radius, $h$ is the Planck constant, $c$ is the vacuum light velocity, and $f_{l u}$ and $n_{l}$ are the absorption oscillator strength and the lower level population number density of the transition, respectively. The normalized line profile $P(\lambda)$ is calculated considering Doppler and Stark broadening that are the dominant mechanisms of spectral line broadening in strongly ionized laserproduced plasmas [99]. Depending on the relative values of Doppler and Stark width, the line shapes are described by Gaussian, Lorentzian, or Voigt profiles. The Doppler width is calculated according to the plasma temperature and atomic mass of the emitting species. For rotational lines of molecular spectra, Stark broadening can be neglected for molecular emission bands observed during the late stage of plume expansion, when the electron density is small (second category in table 2). The population number density of a rotational level $J$ in the vibrational level $v$ of the electronic state $n$ is given by the Boltzmann law

$$
n_{n, v, J}=n_{m o l} \frac{g_{t o t}}{Q_{m o l}} e^{-\left[T_{e}+G_{0}(v)+F_{v}(J)\right] h c / k T}
$$

Here, $n_{m o l}$ and $Q_{m o l}$ are the number density and the partition function of the molecule, and $T_{e}$ and $F_{v}(J)$ are the electronic and rotational terms, respectively. The vibrational term is referred to the lowest vibrational level $G_{0}(v)=G(v)-G(v=0)$. The total statistical weight is given by $g_{t o t}=g_{e} g_{v} g_{J}$, where $g_{e}, g_{v}$, and $g_{J}$ are statistical weights of the electronic, vibrational, and rotational levels, respectively. The statistical weight of the electronic state may be expressed as $g_{e}=(2 S+1)\left(2-\delta_{0, \Lambda}\right)$, where $S$ is the quantum number of the electron spin and $\delta_{0, \Lambda}$ is the Dirac delta function that depends on the component of the electronic orbital momentum along the internuclear axis. The $\delta_{0, \Lambda}$ function characterizes the so-called $\Lambda$-type doubling and we have $\delta_{0, \Lambda}=1$ for $\Lambda=0$ and $\delta_{0, \Lambda}=0$ for $\delta_{0, \Lambda}>0$. The statistical weights of vibrational and rotational levels are $g_{v}=1$ and $g_{J}=g_{N}(2 J+1)$, respectively. The nuclear statistical weight $g_{N}$ accounts for the interaction with the nuclear spin. For heteronuclear molecules such as $\mathrm{AlO}, g_{N}$ is constant and can be ignored in the analysis of the molecular emission spectra. For homonuclear molecules, we have $g_{N}=(2 I+1) /(I+1)$ and $g_{N}=(2 I+1) / I$ for symmetric and antisymmetric states, respectively [28]. Here, $I$ is the quantum number of the atomic nuclear spin. The alternation of the nuclear statistical weight leads to the rotational line intensity alternation observed for the R-branch of the $\mathrm{N}_{2}{ }^{+}$first negative system (see fig. 7). The vibrational and rotational terms $G_{0}(v)$ and $F_{v}(J)$ can be calculated using the term formula given by Herzberg [28] and the partition function is obtained by summing over all states 


$$
Q_{m o l}=\sum_{n} g_{e} \sum_{v} \sum_{J} g_{J} e^{-\left[T_{e}+G_{0}(v)+F_{v}(J)\right] h c / k T}
$$

Assuming complete uncoupling of the rotational, vibrational, and electronic motions, the population number density of a rotational level is given by

$$
n_{n, v, J}=n_{m o l} \frac{g_{\text {tot }}}{Q_{e}\left(T_{e l}\right) Q_{v i b}\left(T_{v i b}\right) Q_{r o t}\left(T_{r o t}\right)} e^{-T_{e} h c / k T_{e l}} e^{-G_{0}(v) h c / k T_{v i b}} e^{-F_{v}(J) h c / k T_{\text {rot }}}
$$

Here, $T_{e l}, T_{v i b}$, and $T_{r o t}$ are the electronic, vibrational, and rotational excitation temperatures, and $Q_{e}$, $Q_{v i b}$, and $Q_{r o t}$ are the electronic, vibrational, and rotational partition functions, respectively. The vibrational and rotational partition functions are computed for the most populated levels being the ground state $(n=1)$ and the lowest vibrational level $(v=0)$. Due to the strong coupling of particles with similar mass (see fig.7), the electronic excitation temperature typically equals the temperature of free electrons in the plasma, whereas the vibrational and rotational temperatures equal the kinetic temperature of heavy particles $T_{h}$. Eq.26 enables calculations with fair accuracy if the population number densities of excited electronic states are small compared to the ground state population number density. Thus, for molecules having a large energy gap between the ground state and the first excited state, the partition function computed by the simplified expression can be used for moderate temperatures. For radicals such as $\mathrm{C}_{2}, \mathrm{AlO}$ or TiO, the energy gap between the ground state and the first excited state is relatively small (see table 1).

Using the superscript' and "for the upper and lower electronic state, respectively, the absorption oscillator strength of a rotational line is given by

$$
f_{J^{\prime}, J^{\prime \prime}}=f_{l u} q_{v^{\prime}, v^{\prime \prime}} \frac{H_{J^{\prime}, J^{\prime \prime}}}{2 J^{\prime \prime}+1}
$$

Here, $f_{l u}$ is the absorption oscillator strength of the electronic transition and $q_{v}, v^{\prime}$ is the Franck-Condon factor for the transition from the vibrational level $v$ ' in the upper electronic state to the vibrational level $v$ " in the lower electronic state. The Hönl-London factor $H_{J, J}$ " can be calculated using the formula given by Kovács [100] for the corresponding type of transition. Inserting the absorption oscillator strength of the rotational line (eq.27) and the corresponding lower level population number density (eq.24 or eq.26) in eq.23, we obtain the absorption coefficient of the rotational line. By summing over all rotational lines and using eq. 22 we compute the spectral radiance of molecular emission from a uniform plasma.

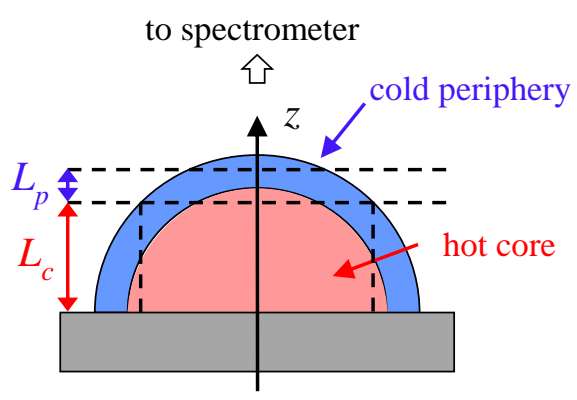

Fig. 10. Scheme of hemispherical ablation plume composed of hot core and cold periphery. 
Plasmas produced by material ablation with ultraviolet nanosecond laser radiation in air or a background gas at atmospheric pressure present a hemispherical shape [101]. The plasma may be characterized in a simplified way by diving it into a hot core with a surrounding cold peripheral zone as illustrated in fig. 10. It was shown that for ablation in argon, the ablation plume is almost uniform in temperature and density [102]. In that case, the cold boundary can be ignored in the spectral analyses can be performed by comparing the measured spectrum to the spectral radiance computed with eq.22. Contrarily, in ambient air, the vapor plume is characterized by the hot core and the cold boundary. In that case, the spectral radiance can be computed by

$$
B_{\lambda}=U_{p}\left(1-e^{-\tau_{p}}\right)+U_{c}\left(1-e^{-\tau_{c}}\right) e^{-\tau_{p}} .
$$

The subscripts $p$ and $c$ stand for the core and peripheral plasma zones, respectively.

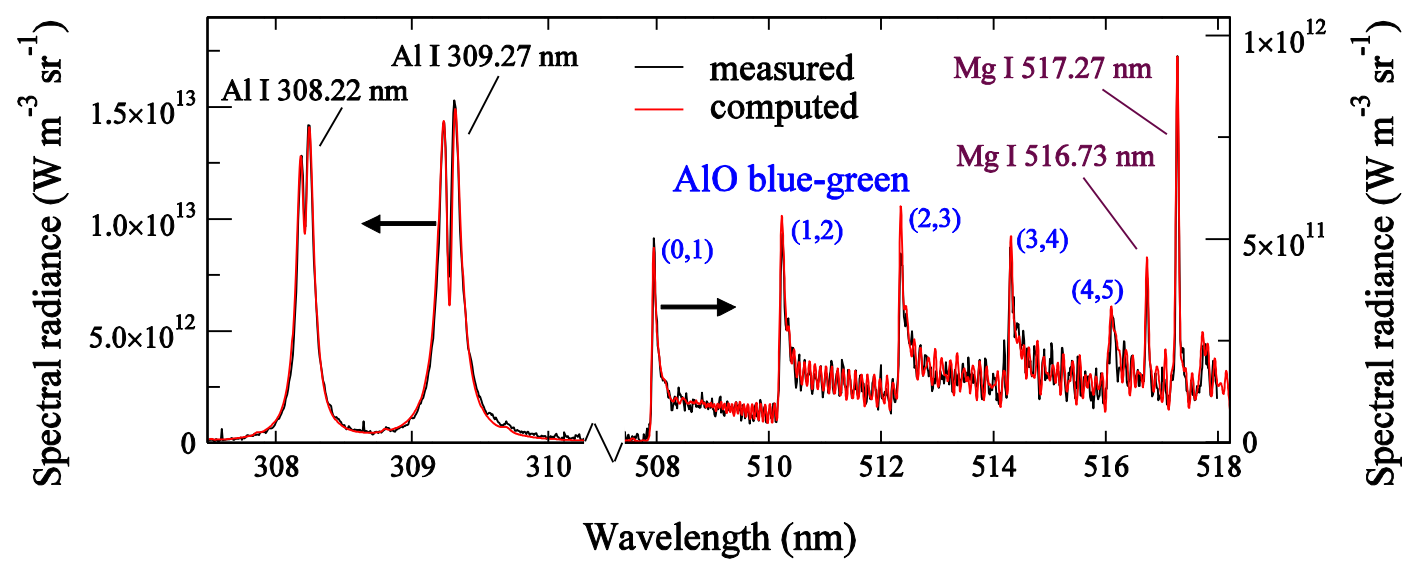

Fig. 11. Aluminum resonance lines and $\Delta v=-1$ vibrational sequence of the AlO blue-green system (see table 1 ) recorded during laser ablation of aluminum in air. Comparison to the spectral radiance of a nonuniform plasma in local thermodynamic equilibrium (see calculation details in Ref. 50).

In fig. 11, we compare the plasma emission spectrum recorded during laser ablation of aluminum in air to the spectral radiance of nonuniform plasma computed using eq.28. The simulation shows that the atomic lines originate from the hot plasma core whereas the molecular emission comes from the cold periphery, where the aluminum resonance lines were re-absorbed. As the cold plume periphery mostly contributes to the atomic emission spectrum through absorption, it can be ignored in calibration-free LIBS analysis as long as optically thin lines are used and interferences with strongly absorbing atomic lines or molecular bands can be avoided. We stress, however, that the extrapolation of the here presented scenario to other chemical reactions is only possible if the involved molecules have dissociation energies similar to that of $\mathrm{AlO}$ (category $\mathrm{B}$ in table 2). Chemical species of larger dissociation energies such as $\mathrm{CO}, \mathrm{CN}$, or $\mathrm{NO}$ (category $\mathrm{A}$ in table 2) may have significant number densities in the hot plasma core and calibration-free analysis of organic materials may fail if chemical reactions are ignored. Compared to metals, ablation of polymer materials is generally characterized by lower initial temperature values and it is not yet clear whether (or to which amount) native bonds are disrupted during the ablation event. The fraction of disrupted bonds certainly depends on the experimental conditions and the conservation of a larger amount of native bonds is expected for femtosecond laser pulse ablation [84] while a large fraction of bonds should be disrupted in typical 
LIBS conditions [85]. However, depending on the material and on the precise irradiation conditions, the question on the role of native bonds remains open. Finally, although several studies illustrate that equilibrium chemistry is appropriate to describe the molecular features observed in the LIBS spectra, the question still remains open for organic materials.

\section{Perspectives.}

In this review we have described in detail the connection between the fundamental aspects of the LIP and the emission spectroscopy. Although many aspects of the emission spectra of the LIP have been elucidated there are several challenges that are still under investigation by the scientific community. As an example, although in the LIP all the elements should emit spontaneous radiation in the same way, as described previously, some elements are very hard to be detected because of their specific electronic configuration. The most important case is the detection of halogen atoms like chlorine and bromine [31]. In these atoms, being the energy difference between the ground state (ns2np5) and the first excited level $(\mathrm{ns} 2 \mathrm{np} 4(\mathrm{n}+1) \mathrm{s})$ more than $7 \mathrm{eV}$, only a small fraction of electrons in the eedf are able to excite this transition, with the results that the population of the starting level for the spontaneous emission is very low. Due to the importance of measuring these elements, several strategies have been proposed: using emission bands of molecules formed by the reaction of the LIP with the surrounding background gas (see previous sections) or performing high UV detection [103]. Another interesting approach is to explore the high ionization degree in the early stage of plasma evolution for detecting the ionic form of the halogen as suggested in Ref. [104]. Another question related to the specific properties of the atoms concerns the ionization energy and the reactivity. In this case the use of theoretical approach could be crucial for the precise interpretation of the plasma characteristics especially when the LIP is produced from the interaction of the laser with fresh and biological samples [105]. Compared to metals or inert gases, the plasmas produced by the ablation of organic materials have been much less investigated. These plasmas are characterized by the formation of diatomic species of large dissociation energy $(\mathrm{CO}, \mathrm{CN}, \mathrm{NH}, \ldots)$, and the chemical processes strongly influence the plasma properties. Due to the poor atomic emission spectra of $\mathrm{C}, \mathrm{N}, \mathrm{H}$ and $\mathrm{O}$, and the complicated features of molecular emission bands, diagnostics of these types of plasma are still challenging. Thus, the question of local thermodynamic equilibrium still remains open. The need of tools for accurate analysis of molecular emission spectra that consider self-absorption and spatial variations is obvious. Another important issue is the application of emission spectroscopy in LIP produced in extreme environment like high pressure atmosphere and liquids [6]. High-pressure LIP spectra is characterized by the degradation of the emission signal due to the increase of density and number of quenching collisions in the plasma and the faster transfer of energy from the plasma to the surrounding environment upon increasing gas pressure. For this reason the spectra are dominated by continuum radiation and the emission lines appear broadened as a consequence of the pressure broadening mechanisms and to the increase of the extent of self-absorption [106]. The most interesting cases, in the frame of extreme environment LIP diagnostics, is the LIP produced in liquids [107], because of several applications, including NPs formation, ocean exploration and various medical applications. As a matter of fact when the plasma expands in liquid environment, as a consequence of the fast transfer of energy from the plasma to the surrounding liquid and to the low compressibility of water, the plasma is quenched faster with respect of gaseous environment and the emission signal is mainly characterized by continuum radiation and short duration [107,108]. Although the continuum radiation can be used, as discussed in section 4 , for the determination of plasma parameters, such like electron temperature and electron number density, the information about the elemental composition in the plasma may be very poor. For this reason in the case of analytical tasks, the double pulse technique 
is usually employed [108]. In this case the first laser pulse induces the cavitation bubble where the second pulse, conveniently delayed from the first one, induces the probing plasma.

This technique allows inducing the plasma produced by the second pulse in the gaseous environment inside the cavitation bubble with the result that the emission spectrum is very similar to the one obtained in an atmospheric gas environment.

Recently the idea of exploiting the continuum radiation as a spontaneous source for the absorption of the elements lines in the plasma is getting a growing interest. This kind of passive absorption allow to detect the resonance transition at the early stage of the expansion when the continuum radiation is very intense and most of the species, as a consequence of the high density effect, are in the low energy levels [46]. This approach can become extremely useful in those experiments where the duration of the plasma is very short, like microchip laser ablation [109] or ablation in high pressure liquids [110]. Although the fundamental aspects of this approach are still not completely elucidated, the phenomenon appears to be very similar to the Fraunhofer effect in astronomical observation. When the plasma expansion starts, the front head of the plasma, that is interacting with the surrounding environment, locally decreases the temperature very fast. Species in low energy states absorb the continuum radiation coming from the core of the high density plasma and produce the absorption spectrum. This approach can be further improved by using a cavity in order to extend the optical path and consequently the sensitivity to specific species [111].

Another question concerns the polarization of continuum plasma radiation. Although the nature of this polarization is still questionable and it is probably due to the reflection of the radiation on the sample surface [112], in principle it may be exploited for decreasing the background radiation in the spectrum [113].

Recent interest in analytical application includes Resonant Laser Ablation, where the same laser pulse provides the ablation and the excitation that induces the fluorescence. In this case the laser has to interact resonantly with the ablated atoms and so a tuneable laser source is required. The corresponding pulse energy does not need to be high like in traditional ablation, because the acceleration of electron should be reduced in order to enhance the excitation for the fluorescence. This technique allows investigating the plasma in the initial stage of expansion, when the number density of ablated material is still very high, and allows to strongly enhance the sensitivity of atomic detection [114].

The list of open questions can be further increased and are out of scope of the present review but they include the role of the particles formed in the plasma or ejected as droplets after the primary ablation, the necessity of improving the accuracy of spectroscopic data such like the spontaneous emission coefficients, the effective correction for self-absorption or matrix effect, the use of NPs for enhancing the ablation efficiency and the plasma excitation [115], the use of magnetic field for confining and shaping the plasma [116], the use of various detection configurations like, for example the use of spatial heterodyne spectroscopy[117].

\section{Conclusion.}

In this review the optical emission of plasma produced after the laser ablation has been discussed, taking into account of the correlation between the plasma fundamental aspects and the observed spectra. In particular, being the LIP a transient plasma, the temporal evolution of the LIP spectrum has been described in details from the atomic and ionic spectra until the molecular spectra. Several information about the features of the LIP spectra has been reported with the aim of giving a a general 
and modern idea of the main issues concerning the emission spectra of the plasmas produced by lasermatter interaction. Finally we reported some novel application of emission spectroscopy for plasma characterization that demonstrates how emission spectroscopy of LIP after several decades is still an hot topic in the contemporary science.

\section{References}

[1] J. L. Shohet, Encyclopedia of Plasma Technology, CRC press 2016.

[2] Radziemski, L.J.; Cremers, D.A., Eds. Laser-inducedPlasma and Applications; Marcel Dekker: New York, 1989

[3] Y.P. Raizer, Laser Induced Discharge Phenomena, Consultants Bureau, New York 1977

[4] Wen S, Mao X, Greif R and Russo R E 2007 J. Appl. Phys. 101023115

[5] A. De Giacomo, M. Dell'Aglio, D. Bruno, R. Gaudiuso, O. De Pascale 2008 Spectrochim. Acta B 63,805 .

[6] A. De Giacomo, M. Dell'Aglio, R. Gaudiuso, S. Amoruso, O. De Pascale 2012 Spectrochim. Acta Part B 781.

[7] A.C. Woods, C.G. Parigger, J.O. Hornkohl 2012 Opt. Lett. 37, 5139-5141.

[8] H.R. Griem, Principles of Plasma Spectroscopy, Cambridge University Press (1997).

[9] C. Aragón, J. A. Aguilera 2008 Spectrochim. Acta B 63, 893.

[10] X.T. Wang, B.Y. Man, G.T. Wang, Z. Zhao, B.Z. Xu, Y.Y. Xia, L.M. Mei, X.Y. Hu 1996 J. Appl. Phys. 80 (3) 1783.

[11] P.R. Wilmott, J.R. Huber 2000 Rev. Mod. Phys. 72315.

[12] A. De Giacomo, V. A. Shakhatov, G.S. Senesi and F. Prudenzano 2002 Appl. Surf. Sci. 186533.

[13] Sibillano, T., Ancona, A., Rizzi, D., Rodil, S.S., Nieto, J.R., Konuk, A.R., Aarts, R., Huis in't Veld, A.J. 2010 Physics Procedia, 5 (PART 2) 429.

[14] C. Phipps, M. Birkan, W. Bohn, H.A. Eckel, H. Horisawa, T. Lippert, M. Michaelis, Y. Rezunkov, A. Sasoh, W. Schall, S. Scharring, J. Sinko 2010 J. Propul. Power 26609.

[15] D. Hahn, N. Omenetto 2010 App. Spectrosc. 64 335A

[16] A.W. Miziolek, V. Palleschi, I. Schechter (Eds.), Laser Induced Breakdown Spectroscopy, Cambridge University Press, New York, 2006.

[17] D. Hahn, N. Omenetto 2012 Appl. Spectrosc. 66 347A.

[18] R.T Wainner, R.S Harmon, A.W Miziolek, K.L McNesby, P.D French, 2014 Spectrochim. Acta Part B 56 (6) 29777.

[19] Spizzichino, V., Fantoni, R. 2014 Spectrochim. Acta - Part B 99201. 
[20] Werheit, P., Fricke-Begemann, C., Gesing, M., Noll, R. 2011 J. of Anal. Atom. Spec. 26 (11) 2166.

[21] A. De Giacomo, C. Koral, G. Valenza, R. Gaudiuso, M. Dell'Aglio 2016 Anal. Chem. 885251

[22] Gimenez, Y., Busser, B., Trichard, F., Kulesza, A., Laurent, J.M., Zaun, V., Lux, F., Benoit, J.M., Panczer, G., Dugourd, P., Tillement, O., Pelascini, F., Sancey, L., Motto-Ros, V. 2016 Scientific Reports, 6, art. no. 29936

[23] Dell'Aglio, M., De Giacomo, A., Gaudiuso, R., Pascale, O.D., Senesi, G.S., Longo, S. 2010 Geochim. et Cosmochim. Acta, 74 (24) 7329.

[24] Craciun V, Craciun D and Boyd I W 1993 Mat. Sci. Eng. B 18178

[25] Hermann J and Dutouquet C 1999 J. Phys. D: Appl. Phys. 322707

[26] Lam J, Amans D, Chaput F, Diouf M, Ledoux G, Mary N, Masenelli-Varlot K, Motto-Ros V and Dujardin C 2014 Phys. Chem. Chem. Phys. 16963

[27] Russo R E, Bol'shakov A A, Mao X, McKay C P, Perry D L and Sorkhabi O 2011 Spectrochim. Acta Part B 6699

[28] Herzberg G 1950 Molecular Spectra and Molecular Structure: Spectra of Diatomic Molecules 2nd edn (London: Chapham and Hall)

[29] Lazic V, Palucci A, Jovicevic S, Poggi C and Buono E 2009 Spectrochim. Acta Part B 641028

[30] Saad S, L’Hermite D and Bousquet B 2014 Spectrochim. Acta Part B 101330

[31] Haisch C, Niessner R, Matveev O I, Panne U and Omenetto N 1996 Fresenius J. Anal. Chem. 356 21

[32] Gaft M, Nagli L, Eliezer N, Groisman Y and Forni O 2014 Spectrochim. Acta Part B 9839

[33] Álvarez C, Pisonero J and Bordel N 2014 Spectrochim. Acta Part B 100123

[34] Forni O, Gaft M, Toplis M J, Clegg S M et al. 2015 Geophys. Res. Lett. 421020

[35] Ferioli F and Buckley S G 2006 Combust. Flame 144435

[36] Casavola A, De Giacomo A, Dell'Aglio M, Taccogna F, Colonna G, De Pascale O and Longo S 2005 Spectrochim. Acta Part B 60975

[37] Grégoire S, Motto-Ros V, Ma Q L, Lei W Q, Wang X C, Pelascini F, Surma F, Detalle V and Yu J 2012 Spectrochim. Acta Part B 7431

[38] López-Moreno C, Palanco S, Laserna J J, De Lucia F Jr, Miziolek A W, Rose J, Walters R A and Whitehouse A I 2006 J. Anal. At. Spectrom. 2155

[39] Abdel-Salam Z, Al Sharnoubi J and Harith M A 2013 Talanta 115422

[40] Samuels A C, DeLucia F C Jr, McNesby K L and Miziolek A W 2003 Appl. Opt. 426205 
[41] Baudelet M, Guyon L, Yu J, Wolf J-P, Amodeo T, Fréjafon E and Laloi P 2006 J. Appl. Phys. 99 084701

[42] Doucet F R, Faustino P J, Sabsabi M and Lyon R C 2008 J. Anal. At. Spectrom. 23694

[43] Serrano J, Moros J and Laserna J J 2016 Phys. Chem. Chem. Phys. 182398

[44] Hermann J, Perrone A and Dutouquet C 2001 J. Phys. B : Mol. Opt. Phys. 34153

[45] Mao X, Bol'shakov A A, Choi I, McKay C P, Perry D L Sorkhabi O and Russo R E 2011 Spectrochim. Acta Part B 66767

[46] De Giacomo A, Dell'Aglio M, De Pascale O, Gaudiuso R, Palleschi V, Parigger C and Woods A 2014 Spectrochim. Acta Part B 100180

[47] Hou H, Chan G C-Y, Mao X, Zorba V, Zheng R and Russo R E 2015 Anal. Chem. 874788

[48] Lam J, Motto-Ros V, Misiak D, Dujardin C, Ledoux G and Amans D 2014 Spectrochim. Acta Part B 10186

[49] Parigger C G, Woods A C, Surmick D M, Gautam G, Witte M J, Hornkohl J O 2015 Spectrochim. Acta Part B 107132

[50] Hermann J, Lorusso A, Perrone A, Strafella F, Dutouquet C and Torralba B 2015 Phys. Rev. E 92 053103

[51] Dutouquet C, Acquaviva S and Hermann J 2001 Spectrochim. Acta Part B 56629

[52] J.A.M. Van der Mullen, Excitation equilibria in plasmas; a classification, Physics Reports of Physic Letter, PRPLCM 191 (2 and 3) (1990) 109-220.

[53] M. Capitelli, F. Capitelli, A. Eletskii 2000 Spectrochim. Acta Part B 55559.

[54] Casavola, A., Colonna, G., De Giacomo, A., Capitelli, M. 2000 J. of Thermophys. and Heat Trans., 17 (2), 225.

[55] A. De Giacomo 2011Spectrochim. Acta B 66661.

[56] H.R. Griem 1963 Phys.Rev. 131 (1963) 1170-1176.

[57] R.W.P. McWhirter, in: R.H. Huddlestone, S.L. Leonard (Eds.), Plasma Diagnostic Techniques, Academic Press, New York, 1965, pp. 201-264, Chapter 5

[58] J.D. Hey 1976 J. Quant. Spectrosc. Radiat. Transfer. 1669.

[59] G. Cristoforetti , A. De Giacomo, M. Dell'Aglio, S. Legnaioli, E. Tognoni, V. Palleschi, N. Omenetto 2010 Spectrochim. Acta B 6586.

[60] Ciucci, A., Corsi, M., Palleschi, V., Rastelli, S., Salvetti, A., Tognoni, E. 1999 Appl. Spectrosc., $53(8) 960$.

[61] Pietanza, L.D., Colonna, G., De Giacomo, A., Capitelli, M. 2010 Spectrochim. Acta Part B 65 (8) 616. 
[62] Morel, V., Bultel, A., Schneider, I., Grisolia, C. 2017 Spectrochim. Acta Part B 1277.

[63] 12. A. F. Fridman, L. A. Kennedy, Plasma Physics and Engineering, 2004, Taylor and Francis, NY.

[64] De Giacomo, A., Gaudiuso, R., Dell'Aglio, M., Santagata A. 2010 Spectrochim. Acta Part B 65 (5) 385 .

[65] Rocchi, F., Molinari, V.G., Mostacci, D., Sumini, M. 2001) Spectrochim. Acta Part B 56 (6) 599.

[66] P. Debye, E. Hückel, On the theory of electrolytes, I, Physik. 24 (1923) 185-206.

[67] H.R. Griem 1962 Phys. Rev. 128997.

[68] G. Cristoforetti, E. Tognoni, L.A. Gizzi, Thermodynamic equilibrium states in laser-induced plasmas: From the general case to laser-induced breakdown spectroscopy plasmas, Spectrochimica Acta Part B 90 (2013) 1-22

[69] J.A. Aguilera, C. Aragon 2004 Spectrochim. Acta Part B 59 (12) 1861.

[70] C. G. Parigger, G. Gautam, D. M. Surmik, 2015 Inter. Rev. of Atom. and Mol. Phys. 6 (1) 43.

[71] M. Burger, M. Skočić, S. Bukvić 2014 Spectrochim. Acta Part B 10151

[72] I. B. Gornushkin, S. V. Shabanov, U. Panne, 2011 J. Anal. At. Spectrom. 26, 1457.

[73] G. Cristoforetti, E. Tognoni 2013 Spectrochim. Acta Part B 79-80 63.

[74] R. Yi, L. Guo, C. Li, X. Yang, J. Li, X. Li, X. Zeng, Y. 2016 J. Anal. At. Spectrom. 31, 961.

[75] Moon, H. Y.; Herrera, K. K.; Omenetto, N.; Smith, B. W.; Winefordner, J. D. 2009 Spectrochim. Acta Part B 64 (7) 702.

[76] C. Aragón, J.A. Aguilera 2010 Spectrochim. Acta Part B 65395.

[77] Itina T E, Tokarev V N, Marine W and Autric M 1997 J. Chem. Phys. 1068905

[78] Colonna G, Casavola A and Capitelli M 2001 Spectrochim. Acta Part B 56567

[79] Hermann J and Dutouquet C 2002 J. Appl. Phys. 9110188

[80] Itina T E, Hermann J, Delaporte P and Sentis M 2002 Phys. Rev. E 066406

[81] Babushok V I, De Lucia F C Jr, Dagdigian P J, Gottfried J L, Munson C A, Nusca M J and Miziolek A W 2007 Spectrochim. Acta Part B 621321

[82] Shabanov S V and Gornushkin I B 2015 Appl. Phys. A 1211087

[83] Nageswara Rao E, Mathi P, Abdul Kalam S, Sreedhar S, Singh A K, Jagatap B N and Venugopal Rao S 2016 J. Anal. At. Spectrom. 31737

[84] Serrano J, Moros J and Laserna J J 2015 J. Anal. At. Spectrom. 302343

[85] Dong M, Mao X, Gonzalez J J, Lu J and Russo R E 2013 Anal. Chem. 852899 
[86] Negre E, Motto-Ros V, Pelascini F and Yu J 2016 Spectrochim. Acta Part B 122132

[87] Vivien C, Hermann J, Perrone A, Boulmer-Leborgne C and Luches A 1998 J. Phys. D: Appl. Phys. 311272

[88] Yip W L, Mothe E, Beldjilali S and Hermann J 2012 J. Appl. Phys. 111063108

[89] Harilal S S, Brumfield B E, Cannon B D and Phillips M C 2016 Anal. Chem. 882296

[90] Radzig A A and Smirnov B M 1985 Reference Data on Atoms, Molecules and Ions (Berlin : Springer)

[91] Harilal S S, Issac R C, Binghu C V, Nampoori V P N and Vallabhan C P G 1996 J. Appl. Phys. 803561

[92] De Lucia F C and Gottfried J L 2013 J. Phys. Chem. A 1179555

[93] Kępa R and Rytel M 1993 J. Phys B : Mol. Opt. Phys. 263355

[94] Hakalla R, Szajna W and Zachwieja M 2012 J. Phys B : Mol. Opt. Phys. 45215102

[95] Balat-Pichelin M, Iacono J and Boubert P 2016 Ceram. Intern. 422761

[96] Dutouquet C and Hermann J 2001 J. Phys. D: Appl. Phys. 343356

[97] Zel'dovich Y B and Y. P. Raizer, Physics of Shock Waves and High Temperature Phenomena 1966 (New York : Academic)

[98] Konjević N and Roberts J R 1976 J. Phys. Chem. Ref. Data 5209

[99] Zhao X Z, Shen L J, Lu T X and Niemax K 1992 Appl. Phys. B 55327

[100] Kovács I 1969 Rotational Structure in the Spectra of Diatomic Molecules (Budapest: Akadémiai Kiadó)

[101] Mercadier L, Hermann J, Grisolia C and Semerok A 2013 J. Anal. At. Spectrom. 281446

[102] Hermann J, Gerhard C, Axente E and Dutouquet C, Spectrochim. Acta, Part B 100189

[103] Gehlen C.D., Wiens E., Noll R., Wilsch G., Reichling K., 2009 Spectrochim. Acta B 64 (10) 1135.

[104] Omenetto N., 2016 N. BDV31-977-32 Final Report, Florida Department of Transportation.

[105] Beldjilali S., Borivent D., Mercadier L., Mothe E., Clair G., Hermann J. 2010 Spectrochim. Acta B 65 (8) 727

[106] D. Böker, D. Brüggemann 2011 Spectrochim. Acta B 6628.

[107] Dell'Aglio M., Gaudiuso R., De Pascale O., De Giacomo A. 2015 Appl. Surf. Sci. 3484.

[108] De Giacomo A., Dell'Aglio M., De Pascale O., Capitelli M. 2007 Spectrochim. Acta B 62 (8) 721. 
[109] Amponsah-Manager K., Omenetto N., Smith B.W., Gornushkin I.B., Winefordner J.D. 2005 J. of Anal. Atom. Spectr. 20 (6) 544.

[110] López-Claros M., Dell Aglio M., Gaudiuso R., Santagata A, De Giacomo A., Fortes F.J., Laserna J.J. 2017 Spectrochim. Acta B in press.

[111] Walsh A.J., Zhao D., Linnartz H., 2013 Rev. Sci. Instr. 84023108.

[112] Asgill M.E., Moon H.Y., Omenetto N., Hahn D.W., 2010 Spectrochim. Acta B 65 (12) 1033.

[113] Penczak J.S., Liu Y., Schaller R.D., Rich D.H., Gordon R.J, 2012 Spectrochim. Acta B 74-75 3.

[114] Wang X., Huang Z., Chu P.C., Cai Y., Leung K.S.Y. ${ }^{c}$, Lum J.T.S., Cheung N.H., 2016 J. Anal. At. Spectrom., 31, 2363.

[115] De Giacomo A., Koral C., Valenza G., Gaudiuso R., Dell’Aglio M., 2016 J. Anal. At. Spectrom. 311566.

[116] Roy, A., Harilal, S.S., Hassan, S.M., Endo, A., Mocek, T., Hassanein, A. 2015 Laser and Particle Beams, 33(2), 175.

[117] Gornushkin I.B., Smith B.W., Panne U., Omenetto N., 2014 Appl. Spectr. 68(9) 1076. 